University of Rhode Island

DigitalCommons@URI

Ocean Engineering Faculty Publications

Ocean Engineering

2000

\title{
Tomographic inversion for sediment parameters in shallow water
}

Gopu R. Potty

University of Rhode Island, gpotty@uri.edu

James H. Miller

University of Rhode Island, miller@uri.edu

James F. Lynch

Kevin B. Smith

Follow this and additional works at: https://digitalcommons.uri.edu/oce_facpubs

\section{Citation/Publisher Attribution}

Potty, G. R., Miller, J. H., Lynch, J. F., \& Smith K. B. (2000). Tomographic inversion for sediment parameters in shallow water. The Journal of Acoustical Society of America, 108, 973-986. doi: 10.1121/1.1286221. Available at: https://doi.org/10.1121/1.1286221

This Article is brought to you for free and open access by the Ocean Engineering at DigitalCommons@URI. It has been accepted for inclusion in Ocean Engineering Faculty Publications by an authorized administrator of DigitalCommons@URI. For more information, please contact digitalcommons-group@uri.edu. 


\section{Tomographic inversion for sediment parameters in shallow water}

Gopu R. Potty, James H. Miller, James F. Lynch, and Kevin B. Smith

Citation: The Journal of the Acoustical Society of America 108, 973 (2000); doi: 10.1121/1.1286221

View online: https://doi.org/10.1121/1.1286221

View Table of Contents: http://asa.scitation.org/toc/jas/108/3

Published by the Acoustical Society of America

\section{Articles you may be interested in}

Inversion for sediment geoacoustic properties at the New England Bight

The Journal of the Acoustical Society of America 114, 1874 (2003); 10.1121/1.1605391

Mid-frequency geoacoustic inversion using bottom loss data from the Shallow Water 2006 Experiment The Journal of the Acoustical Society of America 131, 1711 (2012); 10.1121/1.3666009

Single-receiver geoacoustic inversion using modal reversal

The Journal of the Acoustical Society of America 131, 119 (2012); 10.1121/1.3664083

Geoacoustic inversion in a dispersive waveguide using warping operators

The Journal of the Acoustical Society of America 130, EL101 (2011); 10.1121/1.3611395

Geoacoustic inversion using combustive sound source signals

The Journal of the Acoustical Society of America 124, EL146 (2008); 10.1121/1.2960974

Passive geoacoustic inversion with a single hydrophone using broadband ship noise

The Journal of the Acoustical Society of America 131, 1999 (2012); 10.1121/1.3672688 


\title{
Tomographic inversion for sediment parameters in shallow water
}

\author{
Gopu R. Pottya) and James H. Miller \\ Department of Ocean Engineering, University of Rhode Island, Narragansett, Rhode Island 02882
}

James F. Lynch

Woods Hole Oceanographic Institution, Woods Hole, Massachusetts 02543

Kevin B. Smith

Naval Postgraduate School, Monterey, California 93943

(Received 3 June 1999; accepted for publication 10 April 2000)

\begin{abstract}
This article discusses inversions for bottom geoacoustic properties using broadband acoustic signals obtained from explosive sources. The experimental data used for the inversions are SUS charge explosions acquired on a vertical hydrophone array during the Shelf Break Primer Experiment conducted south of New England in the Middle Atlantic Bight in August 1996. The SUS signals were analyzed for their time-frequency behavior using wavelets. The group speed dispersion curves were obtained from the wavelet scalogram of the SUS signals. A genetic algorithm (GA) was used for the inversion of sound speeds in the water column and compressional wave speeds in the sediment layers. The variations in the sound speeds in the water column were represented using empirical orthogonal functions (EOFs). A range-independent normal mode routine was used to construct the replica fields corresponding to the parameters. Comparison of group speeds for modes 1 to 9 and for a range of frequencies 8 to $200 \mathrm{~Hz}$ was used to arrive at the best parameter fit. An efficient hybrid optimization scheme using the GA and a Levenberg-Marquardt algorithm is presented. Linear perturbation methods were also used to "fine tune" the inversions and to obtain resolution and variance estimates. Analysis was also done to compute the degree of convergence of each of the parameters by explicitly calculating the Hessian matrices numerically. A posteriori estimation of mean and covariance was also done to obtain error estimates. Group speeds for the inverted sound speed fields provide an excellent match to the experimental data. The inverted sediment compressional speed profile compares well with in situ measurements. (C) 2000 Acoustical Society of America. [S0001-4966(00)04907-9]
\end{abstract}

PACS numbers: 43.30.Ma, 43.30.Qd, 43.30.Pc, 41.30.Bp [DLB]

\section{INTRODUCTION}

Acoustic propagation in shallow water is greatly influenced by the properties of the bottom. Calculations of acoustic propagation characteristics based on geotechnical data obtained from cores generally give reliable results. But core surveys are time consuming and may be applicable to only a small area. Hence indirect bottom survey methods which are fast and cover large areas have been developed. Inversions for the properties of the bottom using acoustic data have been given much attention in underwater acoustics. The in situ measurements used as data in these inverse methods are the acoustic field or quantities derived from it. These approaches differ mainly according to the characteristics of the acoustic sources and measurements (travel time, phase, etc.) they utilize for the inversion. Tolstoy et al. ${ }^{1}$ used lowfrequency $(20 \mathrm{~Hz})$ data obtained from air-deployed explosive sources to tomographically estimate environmental parameters in a simulated deep water environment. Ratilal et l. $^{2}$ and Smith $e t a l .{ }^{3}$ used a method based on ambient noise field directivity to invert for the sediment compressional speed. This method estimates a single compressional speed value as

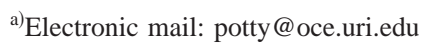

an average for the top layer of sediments. Rapids, Nye, and Yamamoto $^{4}$ designed and tested a 3-D small-scale highresolution cross-well acoustic tomography system in shallow water. A damped least-squared inversion technique was used to construct compressional speed images from measured travel time data.

Tomographic inversions of sediment properties have been performed using both narrow and broadband sources. When a broadband acoustic source is used in a shallow water waveguide, the acoustic propagation exhibits dispersion effects. Group velocity dispersion characteristics have been successfully utilized for the inversion of geoacoustic properties by Lynch, Rajan, and Frisk ${ }^{5}$ using a linear perturbation approach. They have applied this method with success to the data collected during the GEMINI experiment performed in the Gulf of Mexico, using a towed narrow-band acoustic source which output pure tones at 50 and $140 \mathrm{~Hz}$. The perturbation approach breaks down the nonlinear problem into a linear one in the vicinity of the final solution. Hence, an accurate a priori model of the environment is required to achieve good estimates.

Explosives can be used as broadband sources as they have large power output, large bandwidth, and considerable energy at low frequencies. This ensures penetration of acous- 
tic energy deep into the ocean bottom, enabling the determination of ocean bottom parameters to greater depths. The group velocities, i.e., the speeds at which energy is transported, differ for different frequencies and modes. In a shallow water waveguide, high frequencies generally arrive earlier whereas the low frequencies, which interact with the bottom and hence are more important in geoacoustic inversions, arrive later. The dispersion behavior can be used for the inversion of geoacoustic properties of the bottom. It should be noted that the lower frequency data and the higher modes in the dispersion curve should give a better estimate of the sediment features whereas the high-frequency data and lower modes will improve the water column sound speed estimate.

Dispersion effects can be observed by time-frequency analysis of the acoustic pressure signal recorded at sufficiently large distance away from the source. The group speed values are directly extracted from these time-frequency energy distributions. Time-frequency analysis is performed using wavelets. Wavelet analysis examines the frequency distribution of a nonstationary time series using a set of windows that have compact support in time (i.e., decay to zero quickly) and are band limited in the frequency domain. The wavelet transform is a localized transform in both time and frequency and this property can be advantageously used to extract information from a signal that is not possible to unravel with a Fourier or even windowed Fourier transform. The advantage of analyzing a signal with wavelets as the analyzing kernels is that it enables us to study features of the signal locally with a detail matched to their scale, i.e., broad features on a large scale and fine features on a small scale. A detailed description of wavelet analysis can be found in Ref. 6.

In this paper we will emphasize tomographic inversion technique based on these broadband dispersion curves. Nonlinear methods based on exhaustive searches are not constrained in performance by the availability of background information and, thanks to the large increase in computational abilities, are being widely used for inversion in recent times. An inversion scheme for sediment compressional speeds based on a genetic algorithm is used to invert the group speed curves.

The article is organized as follows. The genetic algorithm specifics are introduced in Sec. I. The hybrid inversion scheme is discussed in Sec. II followed by perturbation methods in Sec. II A. Section III covers the procedure for error estimation. The Shelf Break Primer Experiment is described in Sec. IV, followed by data analysis and sensitivity studies in Sec. V, and results and discussions in Sec. VI. The conclusions of the present study and future work planned are presented in Sec. VII.

\section{GLOBAL OPTIMIZATION AND GENETIC ALGORITHMS}

Global optimization schemes such as simulated annealing (SA) and genetic algorithms (GA) have been used increasingly in recent times for the inversion of underwater acoustic signals for bottom properties. Representative references would be Collins and Kuperman, ${ }^{7}$ Hermand and

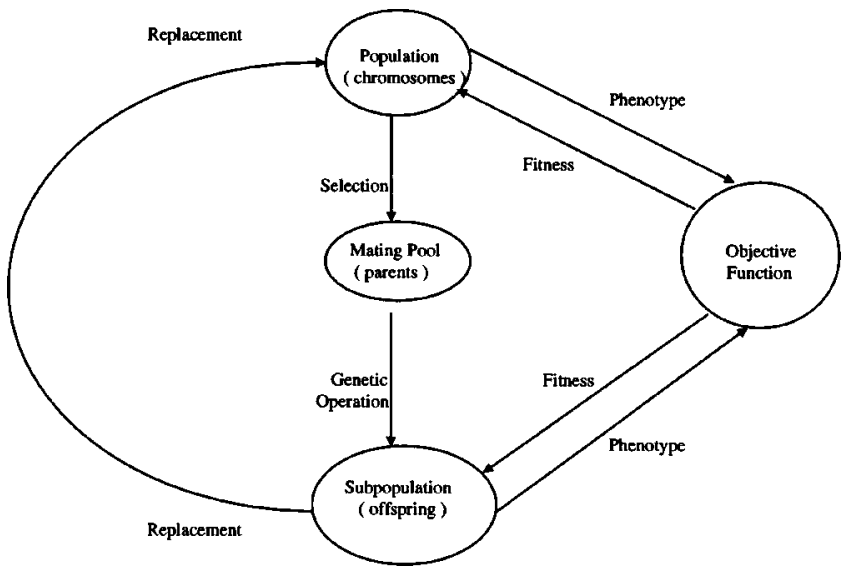

FIG. 1. A typical genetic algorithm cycle (Ref. 9).

Gerstoft, ${ }^{8}$ and Gerstoft. ${ }^{9}$ These methods rely on exhaustive searches, and the time required for the search is often very high. Collins and Kuperman ${ }^{7}$ demonstrated that it is possible to accurately estimate the source location with limited a priori environmental information by expanding the parameter search space of matched field processing (MFP) to include environmental parameters, a method they called "focalization." They used simulated annealing (SA) for searching the large focalization parameter space for optimal parameter values. This scheme was based on matching measured and modeled modal phases.

Genetic algorithms (GAs) are nonlinear optimization schemes, highly efficient in optimizing discontinuous, noisy, highly dimensional and multimodal objective functions. It can be thought of as a "smart" Monte Carlo Search. A GA is not biased by an initial starting model, uses no gradient or curvature information, and has the ability to avoid local minima. Instead of selecting points in model space from a uniform distribution, the points are selected by repeated application of mathematical operators. The search is thereby channeled toward good solutions. Whereas SA is based on a single member meandering in a search space, the GA is based on a population which intercommunicates while meandering in a search space. A simple GA starts with a population of samples randomly generated from the model subspace which is defined by a priori bounds on the model parameters. These search limits are specified initially. The fitness of each member is computed based on the value of the objective function for that member. Then through a set of evolutionary steps the initial population evolves in order to become more fit. An evolutionary step consists of selecting a parental distribution from initial population based on the individual's fitness. The parents are then combined in pairs and operators are applied to them to form a set of children. The operators are traditionally crossover (recombination) and mutation (random bit change) operators. Finally the children replace part of the initial distribution to get a more fit population. The process of going from current to next population constitutes one generation in the execution of a GA. Tang et al. ${ }^{10}$ give a detailed description of these operations. A typical GA cycle is shown in Fig. 1. In the study proposed here a GA will be used to optimize an objective function and 
match measured and modeled modal dispersion characteristics of the broadband acoustic signal. The objective function for the GA inversions, based on minimization of group speed differences, will be modified to incorporate the nonuniform data quality. The objective function is of the form

$$
E(\mathbf{m})=\sum_{i=1}^{N} \frac{\left[d_{i}-F_{i}(\mathbf{m})\right]^{2}}{\sigma_{i}^{2}} ; \quad i=1,2, \ldots, N
$$

where $E(\mathbf{m})$ is the objective function for the $m$ th parameter set and $\sigma_{i}$ is the standard deviation associated with the $i$ th data point. The numerator of Eq. (1) represents the mismatch between the observed data $(\mathbf{d}, N \times 1)$ and the prediction $(F(\mathbf{m}), N \times 1)$ of the forward model. The observed data consists of group speed values calculated using the times of arrival for various modes and frequencies. The predictions are the theoretical group speed values calculated for the sound speed profile constructed using the model parameters.

\section{HYBRID OPTIMIZATION SCHEME}

Using a genetic algorithm does not guarantee that an exact global optimum will be found, even in an infinite amount of time. Because a GA makes no use of gradient or curvature information, it is not clear what type of point in error space the "best model" represents. It could be a local minimum, a global minimum, a saddle point, or none of these. We must also remember that a GA operates in a discrete subspace of the actual model space. For these reasons it is important to closely examine the region around the best model generated by a GA. To overcome these drawbacks many investigators have developed hybrid schemes combining GA with a local search method. Gerstoft ${ }^{11}$ suggested a combination of global GA and a local Gauss-Newton method. Taroudakis and Markaki ${ }^{12}$ proposed another hybrid scheme wherein the reference environment defined using matched field processing with a GA is subsequently used in connection with a modal phase inversion scheme. This linear modal inversion is meant to fine tune the results obtained through the matched field tomography. We use the optimum parameters obtained from the genetic algorithm inversion as the starting point for a local search using the LevenbergMarquardt algorithm, which is more robust than the GaussNewton method. This algorithm uses a search direction which is a cross between the Gauss-Newton direction and the steepest descent direction. By applying this method at the end of the GA search, we can assess the quality of the GA solution locally and perhaps find a better solution.

\section{A. Broadband perturbative modal inversion}

Linear inversion methods give good estimates of the environmental parameters when good a priori knowledge of the environment is available. Lynch, Rajan, and Frisk ${ }^{5}$ have successfully used a linear perturbative approach for the inversion of group velocity data for the sediment sound speed. The expression which relates the group velocity dispersion curve to the bottom sound speed profile is

$$
\begin{aligned}
\frac{1}{v_{m}^{g}}-\frac{1}{v_{n m}^{g(0)}} & \\
= & \left(\frac{1}{k_{m}}\right) \int_{0}^{\infty}\left\{\left[2 \omega_{0}^{-1} \rho^{-1}(z)\left|\psi\left(\omega_{0}, z\right)\right|^{2} k^{2}(z) \frac{\Delta c(z)}{c(z)}\right]\right. \\
& +\left[\left(\frac{1}{k_{m} v_{m}^{g(0)}}\right) \rho^{-1}(z)\left|\psi\left(\omega_{0}, z\right)\right|^{2} k^{2}(z) \frac{\Delta c(z)}{c(z)}\right] \\
& \left.+\left[\rho^{-1}(z) \frac{d}{d \omega}\left|\psi\left(\omega_{0}, z\right)^{2}\right| k^{2}(z) \frac{\Delta c(z)}{c(z)}\right]\right\} d z,
\end{aligned}
$$

where $v_{m}^{g}$ is the experimentally measured group velocity for the $m$ th mode at $\omega_{0}, v_{m}^{g(0)}$ is the group velocity of the $m$ th mode at $\omega_{0}$ for the background model, $k_{m}$ is the $m$ th eigenvalue for the background model, $\rho(z)$ is the density profile for the background model, $\psi\left(\omega_{0}, z\right)$ is the normalized mode function for the background model for the $m$ th mode at $\omega_{0}$, $c(z)$ is the background sound speed profile, $\Delta c(z)$ is the perturbation in sound speed from the background profile, and $k(z)$ is $\omega / c(z)$, where $\omega$ is the angular frequency.

Equation (2) is a Fredholm integral equation of the first kind which can be written as a discrete sum

$$
d_{i}=\sum_{j} G_{i j} q_{j}, \quad i=1,2, \ldots, N ; \quad j=1,2, \ldots, M,
$$

or, in matrix form,

$$
\mathbf{d}=\mathbf{G q} \text {, }
$$

where $\mathbf{d}$ is an $N \times 1$ data vector, $\mathbf{q}$ is an $M \times 1$ vector of unknown perturbations, and $\mathbf{G}$ is an $N \times M$ kernel matrix. The data vector consists of the differences between the measured group slowness and the group slowness for the background profile [left-hand side of Eq. (2)] and the unknowns are the perturbations in sound speed $[\Delta c(z)]$. Thus this linear tomographic system is characterized by an $N \times M$ Frechet matrix (G) which is a linear mapping from the $M$-dimensional parameter space to the $N$-dimensional data space. The above linear problem can be solved by singular value decomposition. This is discussed in detail by Lynch et $a .^{5}$ To incorporate the nonuniform data quality Parker ${ }^{13}$ suggested weighting the data with standard errors. Weighted data with unit variance is obtained by scaling the data by the standard errors

$$
\mathbf{e}=\mathbf{\Sigma}^{-1} \mathbf{d}
$$

where $\mathbf{d}$ is the data vector, $\mathbf{\Sigma}$ is a diagonal matrix with $\sigma_{1}, \sigma_{2}, \ldots, \sigma_{N}$ as the diagonal elements, and $\sigma$ 's are the standard deviations associated with data. In our application these $\sigma$ 's can be approximately estimated from the time-frequency distributions based on the spread of the spectral lines. Multiplying both sides of Eq. (4) by $\boldsymbol{\Sigma}^{-1}$ and using Eq. (5),

$$
\mathbf{e}=\boldsymbol{\Sigma}^{-1} \mathbf{G W y}
$$

where

$$
\mathbf{y}=\hat{\mathbf{R}} \mathbf{q}
$$

and $\mathbf{G}$ is the kernel matrix, $\mathbf{W}$ is the diagonal matrix of weights of a numerical quadrature approximation (trapezoi- 


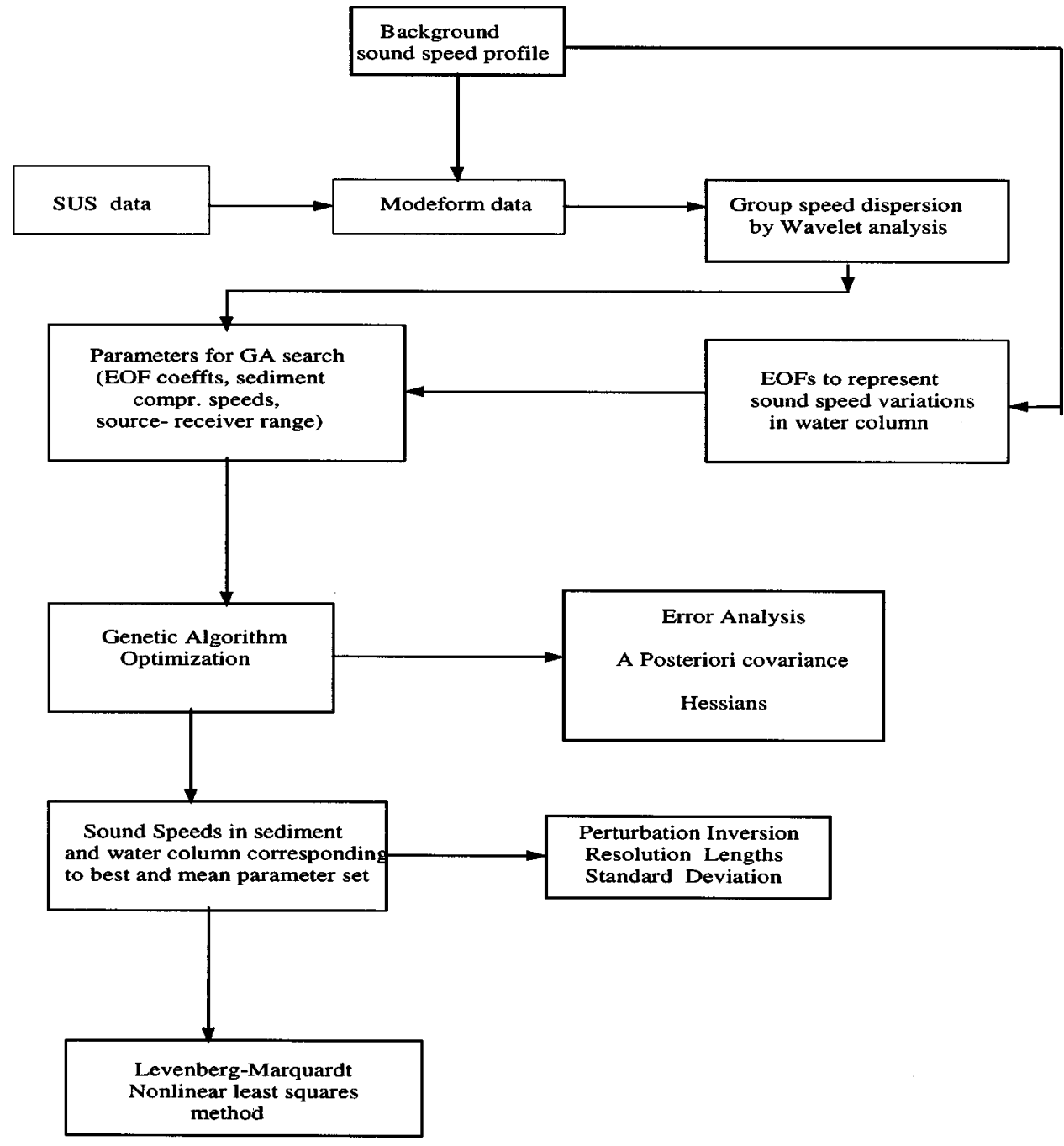

FIG. 2. Schematic representation of the steps involved in the inversion scheme.

dal rule in the present case), $\hat{\mathbf{R}}$ is the regularizing matrix, and $\mathbf{q}$ are the unknowns of the original problem $[\Delta c(z)] . \mathbf{W}$ is introduced here to stabilize the numerical implementation. For $L_{2}$ norm minimization $\hat{\mathbf{R}}$ can be taken as $\mathbf{W}^{1 / 2}$. These steps are discussed in detail by Parker. ${ }^{13}$

\section{ERROR ESTIMATES AND VALIDATION OF THE INVERSION RESULTS}

The goals of most inversions include not only finding a model which best fits the data, but also estimating error bounds on the parameters. Even though these error bounds cannot be considered as validation of the inversion, they do provide useful indication about the quality of the inversions. We have quantified the errors in our current work using different approaches based on different criteria. A posteriori covariance is estimated by defining an a posteriori model probability. Local error bounds of the model parameters are estimated using numerically evaluated Hessian matrices. Elements of the Hessian matrix are the second partial derivatives of the objective function with respect to the model parameters. They were evaluated numerically in the neighborhood of the best solution. In addition, resolution and variance estimates are also obtained using the linear perturbation approach.
In the present study the compressional speeds generated using the GA inversion are used as a background profile for the perturbation inversion. Thus the GA inversion is further refined using linear perturbation methods, as well as the Levenberg-Marquardt optimization method. The entire inversion process is schematically represented in the block diagram shown in Fig. 2.

\section{A. A posteriori probability density and model covariance}

During the GA optimization procedure, all the samples of the model space are stored and then later used to estimate a posteriori probabilities. From a Bayesian point of view, the solution of the inverse problem can be characterized by an $a$ posteriori probability distribution of the model parameters. Hence, in addition to the best possible estimate, moments of the a posteriori distributions such as mean and covariance can also be calculated. Based on an analogy with SA, Sen and Stoffa ${ }^{14}$ and Gerstoft ${ }^{9}$ have used the Gibbs probability distribution to define the a posteriori probability density in the model space. The Gibbs probability $\sigma(\mathbf{m})$ is given by

$$
\sigma(\mathbf{m})=\frac{\exp [E(\mathbf{m}) / T]}{\sum \exp [E(\mathbf{m}) / T]},
$$


where $E(\mathbf{m})$ is the fitness function or equivalently the value of the objective function for the model vector $\mathbf{m}$. Here $T$ is a control parameter similar to temperature used in SA and the sum is taken over all derived models. Choosing the energy of the fittest in the sample as the value of $T$ will favor the fittest part of the population. Instead if the energy of the least fit sample is chosen as the value of $T$, a more even weighting of the population can be obtained. Experience has shown that a good temperature is the average of the best 50 samples. ${ }^{15} \mathrm{We}$ can estimate the mean and model covariance matrix by defining

$$
\overline{\mathbf{m}}=\int \mathbf{m} \sigma(\mathbf{m}) d \mathbf{m},
$$

which can be approximated by the sum

$$
\overline{\mathbf{m}}=\Sigma \mathbf{m} \sigma(\mathbf{m}) .
$$

The covariance matrix can be obtained as

$$
\mathbf{C}_{\mathbf{m}}=\int(\mathbf{m}-\overline{\mathbf{m}})(\mathbf{m}-\overline{\mathbf{m}})^{T} \sigma(\mathbf{m}) d \mathbf{m} .
$$

This can also be written as

$$
\mathbf{C}_{\mathbf{m}}=\int \mathbf{m} \mathbf{m}^{T} \sigma(\mathbf{m}) d m-\overline{\mathbf{m}} \overline{\mathbf{m}}^{T}
$$

or as the sum

$$
\mathbf{C}_{\mathbf{m}}=\sum \mathbf{m} \mathbf{m}^{T} \sigma(\mathbf{m})-\overline{\mathbf{m}} \overline{\mathbf{m}}^{T} .
$$

The square roots of the diagonal terms of $\mathbf{C}_{\mathbf{m}}$ are the standard deviations or the error bars of the model parameters from the mean. Similarly, the off-diagonal terms show how the different model parameters influence each other.

The model parameter values and their probabilities are stored for all the generations in the GA run. At the end of the GA run all the probability values are summed and each value is divided by the sum to derive the normalized a posteriori probability densities. Using this, the a posteriori mean and covariance matrix are evaluated.

We can also examine the locally defined error bounds on the model parameters by computing the covariance matrix using the Hessian matrix. When the data are uncorrelated and Gaussian, the covariance matrix can be computed using the following equation: ${ }^{16}$

$$
\left[\mathbf{C}_{\mathbf{m}}\right]_{\text {local }}=\sigma_{\mathbf{d}}^{2}\left[\frac{1}{2} \frac{\partial^{2} E}{\partial \mathbf{m}^{2}}\right]_{\mathbf{m}=\mathbf{m}_{\text {est }}}^{-1},
$$

where $\sigma_{\mathbf{d}}$ is the standard deviation of the error in the data. The variance of the model error is the diagonal of $\mathbf{C}_{\mathbf{m}}$. Thus the uncertainty in a set of model parameters is the product of the uncertainty in the data and the second-order curvature of the error space about the point $\mathbf{m}_{\text {est }}$. It should be noted that by sampling a much larger model space, the GA gives a more realistic estimate of error bounds than this local method. We have evaluated error estimates using both methods and they seem to agree with each other for our particular case.

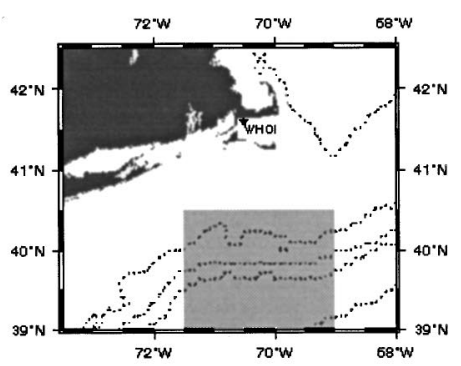

PRIMER III Field Study July--August, 1996

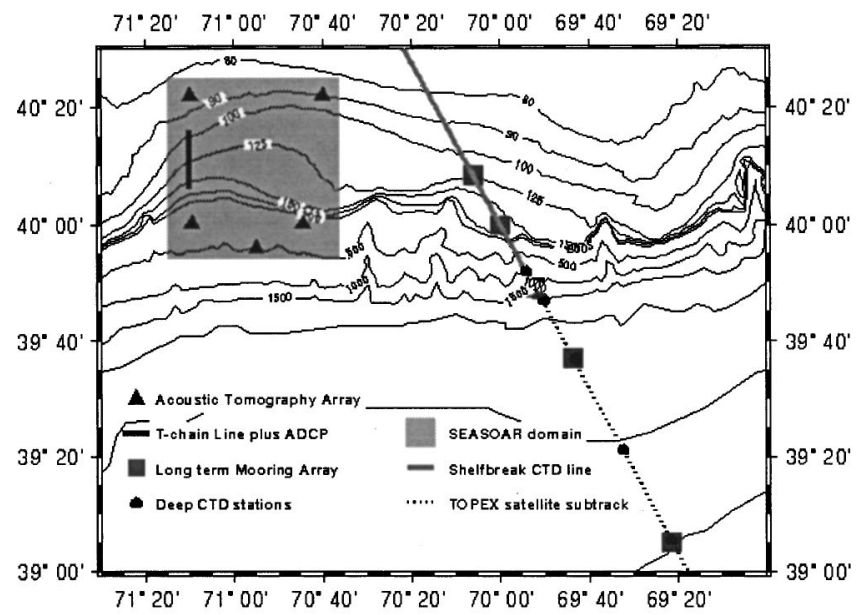

FIG. 3. Location of Shelf Break Primer Experiment. Locations of VLAs and the tomographic sources are also shown.

The model resolution length and standard deviation can be calculated based on the linear perturbation inversion using the singular value decomposition approach. This is described in detail by Lynch et al. ${ }^{5}$

\section{SHELF BREAK PRIMER EXPERIMENT}

In the 1996-1997 Shelf Break Primer Experiment a number of oceanographic and acoustic measurements on the shelf break south of New England in the Middle Atlantic Bight during summer and winter conditions were conducted. Oceanographic observations mainly consisted of SeaSoar ${ }^{17}$ hydrography, shipboard acoustic Doppler current profile (ADCP) measurements, and air-deployed expendable bathy thermographic (AXBT) drops. Some of these measurements were used in this study as background environmental information. Figure 3 shows the location of the experimental site and the positions of the vertical line arrays (VLA's), acoustic tomographic sources, and bathymetry. The acoustic component involved transmissions from moored tomographic sources and explosive SUS charges. The SUS component of the experiment involved the acquisition of broadband acoustic data on two vertical line arrays located on the continental shelf on the northwest and northeast corners of the experimental area, in water depths of approximately $90 \mathrm{~m}$. A P-3 aircraft from the Naval Air Warfare Center in Patuxent Naval Air Station dropped over 80 MK61 explosive charges in an inverted F-pattern during both summer and winter cruises. Figure 4 shows the SUS drops along the slope and the loca- 


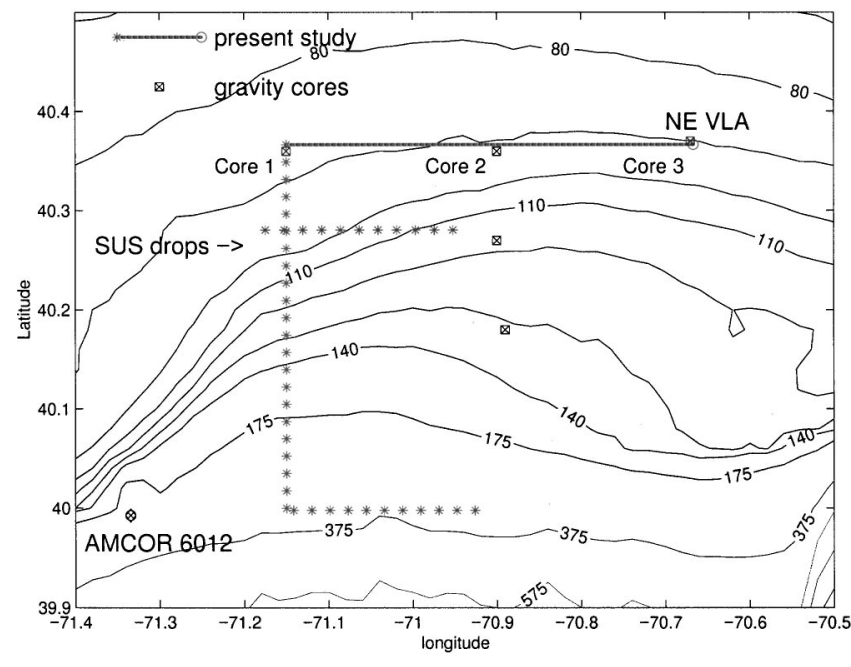

FIG. 4. The SUS drop locations at the experimental site. The AMCOR site is shown in the lower left corner of the figure. The propagation path corresponding to the present study is also shown. The gravity cores on this path (cores $1-3$ ) are used in this study for the comparison and validation of the inversion.

tion of the Atlantic Margin Coring (AMCOR) program site 6012. Sediment compressional sound speeds at various depths were calculated at this AMCOR location using the Biot-Stoll model and then compared with the inverted values. The SUS charges were dropped at a spacing of one nautical mile. The SUS drop effort provides a temporal "snapshot" over a larger spatial area and a larger acoustic bandwidth than conventional ocean tomography measurements. Thus these shots complement the electronic tomography sources deployed in the experiment. MK61 SUS charges are $1.8 \mathrm{lbs}$ of TNT and were set to detonate at a depth of 18 $\mathrm{m}$ in water depths varying from 80 to $300 \mathrm{~m}$.

\section{A. Geoacoustic data at the PRIMER site}

The nature of the seafloor sediments in the continental shelf and upper slope regions of the Middle Atlantic Bight have been studied by various investigators (Knebel and Spiker, ${ }^{18}$ Robb et al.,${ }^{19}$ Poag, ${ }^{20}$ and Hatheway et al. $){ }^{21}$ Analyses of cores taken from this area reveals nearly horizontal layers of Pleistocene and early Holocene silty clays covered by various thicknesses of Holocene sands from 1 to $20 \mathrm{~m} .{ }^{18}$ In a location southeast of Primer site $\left(73^{\circ} \mathrm{W}, 39^{\circ} \mathrm{N}\right)$ close to shelf break they estimated the average thickness of the surface sand layer to be $5 \mathrm{~m}$ using seismic reflection profiles and core samples. The sediment below the surface layer was texturally diverse and consisted of silty and clayey sands, sandy and silty clays, sand-silt-clays and clays. The clay layers are poorly sorted and very stiff. Layers containing more than $75 \%$ sand are also present, but they are intercalated with muddy zones. Gravel is also found in some of the cores. In another location at the upper continental slope $\left(72^{\circ} 50^{\prime} \mathrm{W}, 38^{\circ} 50^{\prime} \mathrm{N}\right)$, Robb et al. ${ }^{19}$ observed a thin surface layer (generally less than $2 \mathrm{~m}$ thick) of medium-grained Holocene sediments underlain by texturally diverse Pleistocene sediments composed of silty clay, silty sand, clayey sand, or sandy clay. The thickness of the surface Holocene sediment
TABLE I. Geoacoustic data at the AMCOR-6012 site (Ref. 24). These parameters were used for estimating sediment compressional speeds using Biot-Stoll theory.

\begin{tabular}{lll}
\hline \hline \multicolumn{1}{c}{ Parameter } & \multicolumn{1}{c}{ Unit } & \multicolumn{1}{c}{ Value } \\
\hline Gain density & $\mathrm{kg} / \mathrm{m}^{3}$ & 2600 \\
Fluid density & $\mathrm{kg} / \mathrm{m}^{3}$ & 1000 \\
Grain bulk modulus & $\mathrm{Pa}$ & $3.6 \times 10^{10}$ \\
Fluid bulk modulus & $\mathrm{Pa}$ & $2.25 \times 10^{9}$ \\
Fluid viscosity & $\mathrm{kg} / \mathrm{m} . \mathrm{s}$ & $1 \times 10^{-3}$ \\
Shear specific loss & & 0.02 \\
Volumetric specific loss & & 0.015 \\
Added mass coefficient & & 0.2 \\
Mean grain diameter-silty clay & $\mu \mathrm{m}$ & 2.7 \\
\hline \hline
\end{tabular}

layer consisting of medium-to coarse-grained sand reaches 20 -m thickness along the outer New Jersey shelf. ${ }^{20}$ Poag also estimated the maximum thickness of the Pleistocene layer, consisting of clayey and silty sands and silty clays, to be 170 $\mathrm{m}$ in the same location (approximately $39^{\circ} \mathrm{N}, 73^{\circ} \mathrm{W}$ ). Hatheway et l. $^{21}$ also reports Pleistocene layers consisting of silty clay and fine sand with occasional layers of pea gravel in the same location. On the upper continental slope, thick Pleistocene sequences consisting of silty and sandy clays ${ }^{20}$ silty clay units, alternating with silty sands and fine sands at some locations ${ }^{21}$ were found. Over $50 \%$ of the clay sediments in the region have an effective stress friction angle of approximately 30 degrees, ${ }^{21}$ which corresponds to silty sands or inorganic silt. The Pleistocene clays were mostly inorganic and have medium plasticity.

Broacher and Ewing 22 used high-resolution seismic reflection data to estimate seismic velocities in an area on the continental shelf south of Long Island, NY, in approximately $64 \mathrm{~m}$ of water. Sediment samples collected near this experimental site recovered coarse to fine sand. The top $30 \mathrm{~m}$ of sediments consisted of Pleistocene and younger sediments. They estimated the thickness of the Pleistocene sediments as $31 \mathrm{~m}$ and a velocity of $1750 \mathrm{~m} / \mathrm{s}$. It should be noted that the uncertainty associated with these measurements is high (450 $\mathrm{m} / \mathrm{s}$ ). McGinnis and Otis ${ }^{23}$ obtained similar results using refracted arrivals in shelf areas of George's Bank and Long Island. They reported a mean velocity in the upper few tens of meters of sediments at locations close to $70^{\circ} \mathrm{W}, 40^{\circ} \mathrm{N}$ of the order of $1650-1675 \mathrm{~m} / \mathrm{s}$. They also estimated the vertical velocity gradients at this location to be $1.2 \mathrm{~m} / \mathrm{s} / \mathrm{m}$.

Even though the areas adjacent to the Primer site were well investigated, very little geoacoustic data is available about the near surface sediments (top 1 to $100 \mathrm{~m}$ ) within the present experimental location. The only published core data reported is from the AMCOR-6012 project. During 1976 the U.S Geological Survey conducted the Atlantic Margin Coring Project (AMCOR) to obtain information on the geotechnical and other properties at sites widely distributed along the continental shelf and slope of the Eastern United States. ${ }^{21}$ The analysis of the cores obtained during this project provided information on the porosity, bulk density, and other geotechnical parameters down to a depth of about $300 \mathrm{~m}$ below seafloor. ${ }^{24}$ The AMCOR-6012 site, at $39^{\circ} 59.57^{\prime} \mathrm{N}$ and $71^{\circ} 20.09^{\prime} \mathrm{W}$, is near the southwest corner of the experimental area at a water depth of $263 \mathrm{~m}$. The location of the drill 

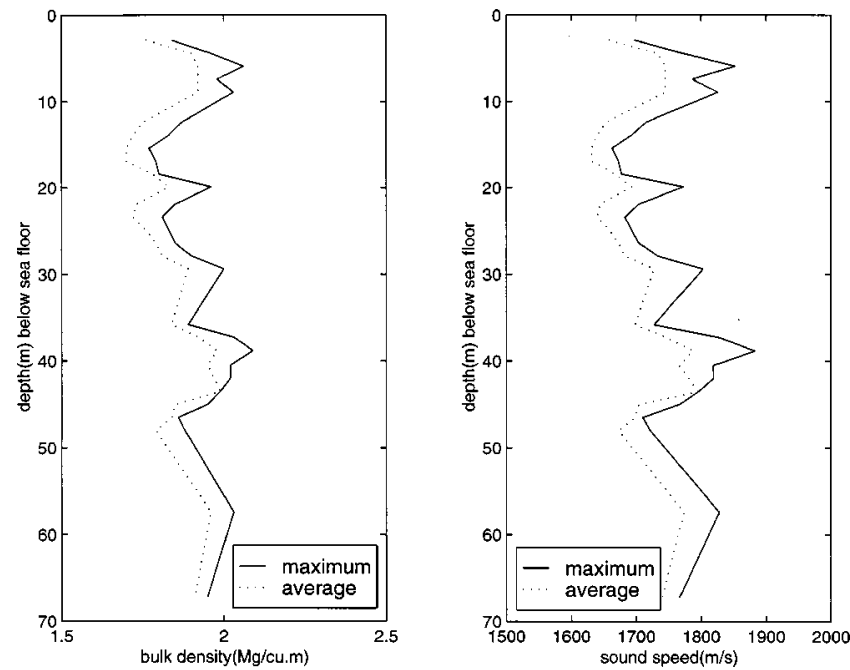

FIG. 5. Compressional sound speed at AMCOR 6012 site. The two curves show the maximum and average bulk densities and the compressional sound speeds calculated using them.

site in relation to the SUS drop locations is shown in Fig. 4. Bulk densities at various depths and the compressional wave speeds calculated using the geotechnical parameters (Table I) based on the Biot-Stoll model ${ }^{25}$ are shown in Fig. 5. The two profiles represent the average and maximum values of bulk density and the corresponding compressional speeds calculated based on them. In the absence of any error estimates these two curves can be used to gauge the spread of the values at each depth. The deviation from the mean is of the order of $30 \mathrm{~m} / \mathrm{s}$ except at layers with high compressional speeds where it is nearly $75 \mathrm{~m} / \mathrm{s}$. Trevorrow and Yamamoto $^{26}$ obtained the compressional wave speed profile at the AMCOR 6010 site using gravity wave inversion technique. AMCOR 6010 is located south west of the Primer site in the outer continental shelf in approximately $69 \mathrm{~m}$ of water depth. It is interesting to note that the highest speed close to the surface (approximately $1800 \mathrm{~m} / \mathrm{s}$ ) is at $5-\mathrm{m}$ depth for AMCOR 6012 whereas it is at $30 \mathrm{~m}$ for AMCOR 6010.

Since direct measurements of geoacoustic parameters were scarce in the experimental area, five gravity cores were taken to compare with the inversions. These five cores were taken at locations indicated in Fig. 4. The maximum core penetration in the shelf locations was approximately $1.5 \mathrm{~m}$ whereas it was $1.0 \mathrm{~m}$ at deeper slope locations. At these depths, core penetration was limited by the presence of sand. The core samples were analyzed at the Marine Geomechanics Laboratory in University of Rhode Island using a multisensor core logger. This analysis provided profiles of compressional speed, bulk density, and porosity. Compressional speeds obtained from cores which lie on the propagation path (cores 1-3) were used for comparison and validation of inversion. The average compressional speed for the top $1.5 \mathrm{~m}$ of sediments is of the order of $1575-1600 \mathrm{~m} / \mathrm{s}$.

\section{ANALYSIS OF PRIMER SUS DATA}

Data from the SUS explosions were collected on two VLAs at the northwest (NW) and northeast (NE) corners of the experimental area during the PRIMER experiment. The positions of these VLAs and the SUS drop locations are

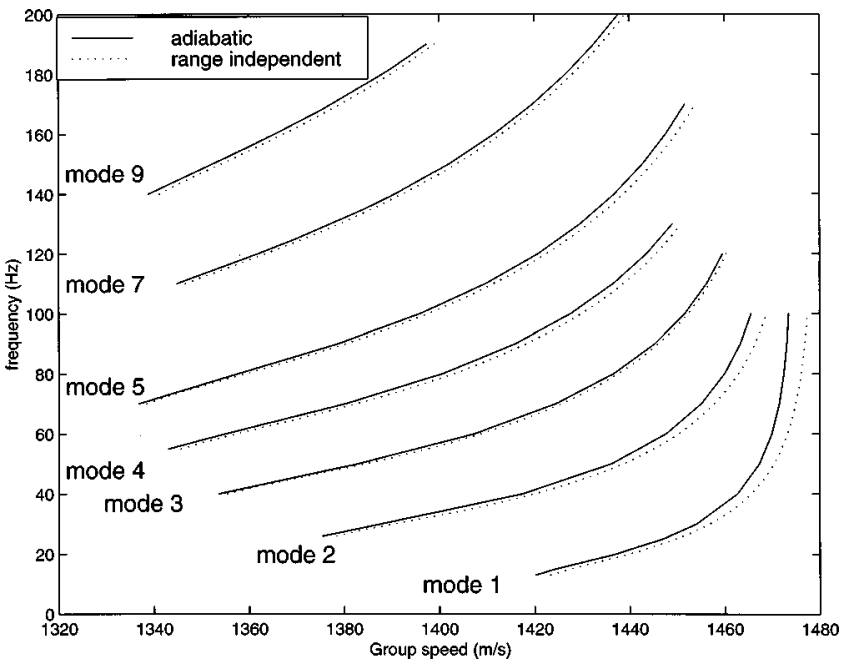

FIG. 6. Effect of range dependence of ocean sound speeds on group speeds.

shown in Fig. 3. Data were collected at a sampling frequency of 1395.09 samples/second at the NE VLA, whereas the sampling frequency was $3906.25 \mathrm{samples} / \mathrm{second}$ at the NW array. The NE VLA consisted of 16 hydrophones in water depths varying from 45.4 to $93 \mathrm{~m}$. The propagation path from the northernmost three SUS explosions to the NE array is over a fairly uniform bathymetry and was chosen for the present study. Longitudinal peak-to-peak variability in the sound speeds in the water column is of the order of $5 \mathrm{~m} / \mathrm{s}$ and bathymetric variations are of the order of $3 \mathrm{~m}$. Influences of range dependence of bathymetry and ocean sound speed are not significant. Hence range-independent conditions were assumed for the propagation. This assumption was verified by comparing the group speed values calculated assuming range independence and also using adiabatic range dependence (Fig. 6).

Figure 7 shows the time series from the SUS explosion at the NW corner received at the top hydrophone of the NE VLA. The signal is broadband in nature and dispersion effects are evident in the time series, e.g., separation of the

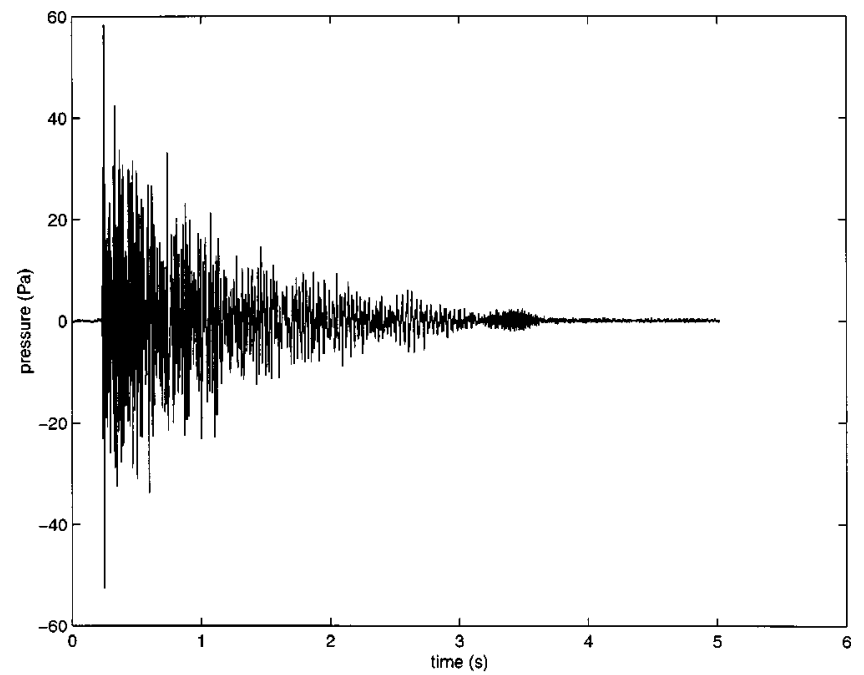

FIG. 7. Time series of SUS signal received at $40 \mathrm{~km}$ on one of the hydrophones. The arrival times are arbitrary. Note the arrival of a mode at high frequency at $3.3 \mathrm{~s}$. 


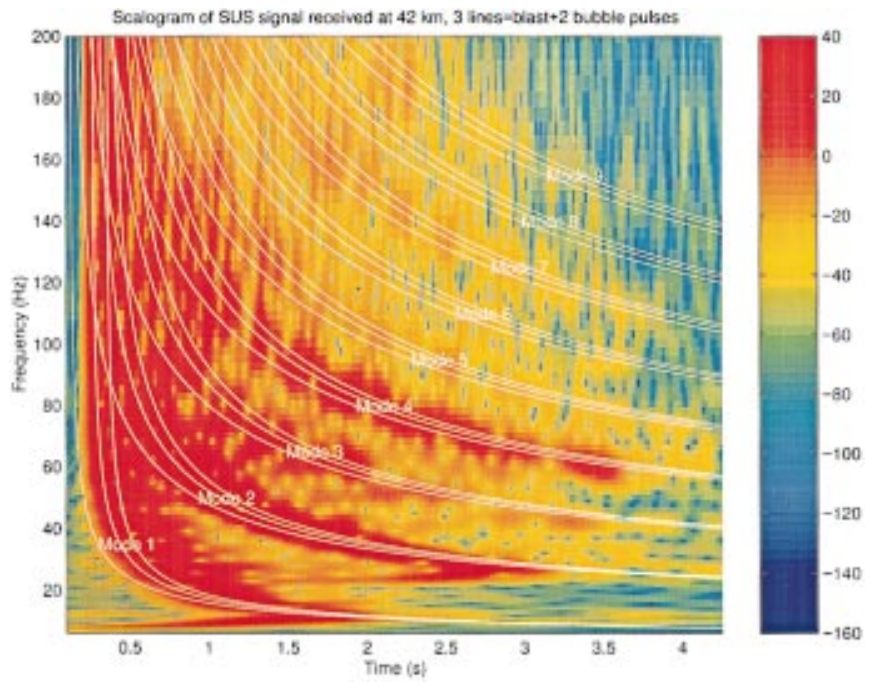

FIG. 8. Time-frequency scalogram of SUS signal shown in Fig. 7 using Morlet wavelet. The color scale is arbitrary and corresponds to intensity in decibels. The three lines correspond to the blast and two bubble pulses.

times of arrival of various frequencies is apparent. This signal is then further processed using a Morlet wavelet. A standard wavelet package ${ }^{27}$ was used for this analysis. Following the shock wave due to the initial blast, a series of positive pressure ("bubble") pulses are emitted by the pulsating gas globe at the successive instants of minimum volume. The time of arrival between the direct blast and the first bubble pulse is approximately $0.12 \mathrm{~s}$ for $1.8 \mathrm{lbs}$ of TNT exploding at $18-\mathrm{m}$ depth. ${ }^{28}$ Because of the presence of these bubble pulses, it is difficult to identify and separate early individual mode arrivals. Late arrivals are better separated, and are easily identified as can be seen in Fig. 8. The continuous lines in Fig. 8 represents the theoretical curves for the direct blast and the two bubble pulses. These theoretical lines are generated assuming a simple sound speed model based on the available historical data. These theoretical group speed curves help to identify the individual mode arrivals especially when multiple peaks are present corresponding to a mode arrival. The arrival times are calculated based on Fig. 8 and the arrival pattern for individual frequencies as shown in Fig. 9 for $40 \mathrm{~Hz}$. Another difficulty encountered in obtaining mode arrival times is the poor resolution at higher frequencies which is inherent in our wavelet analysis. Although the wavelet analysis gives better time-frequency resolution than the short time Fourier transform (STFT), the resolution is not uniform across the entire time-frequency plane. Specifically, it does not give good frequency resolution at higher frequencies when compared to low frequencies. Hence it is very difficult to extract group speed values for higher modes at higher frequencies.

The peak intensities are located for representative frequencies and the corresponding arrival times are used for calculating group speeds. The standard errors of the data at various frequencies and modes are estimated from the width of the peaks. The intensity of the acoustic signal at $40 \mathrm{~Hz}$ is shown in Fig. 9. The peaks corresponding to modes 1-3 are at $0.6,1.5$, and $3.4 \mathrm{~s}$, respectively. The distance between points corresponding to $95 \%$ of peak intensity is taken as an

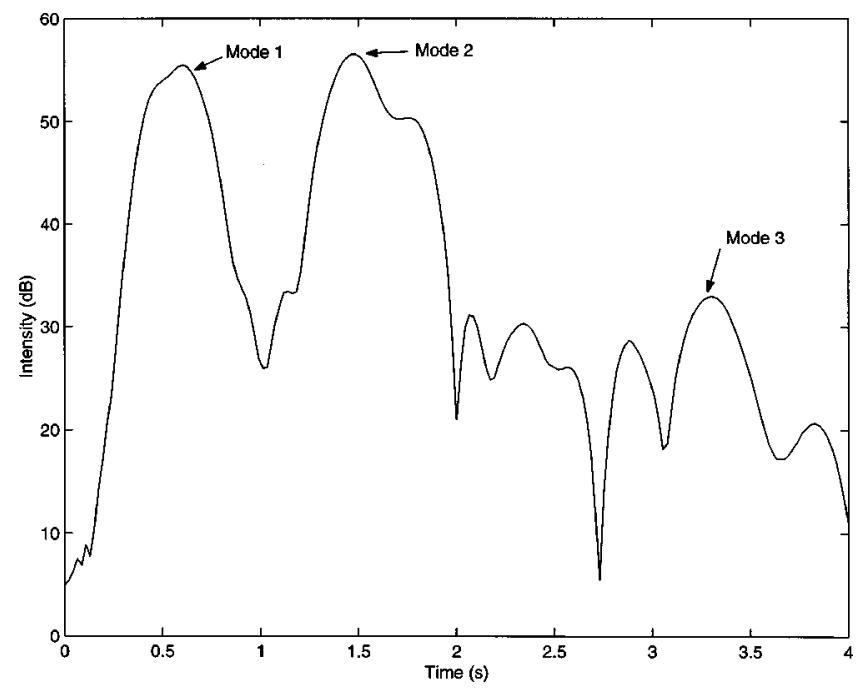

FIG. 9. The acoustic intensity at $40 \mathrm{~Hz}$ showing the time of arrivals corresponding to modes $1-3$. Mode 3 intensity is very small compared to modes 1 and 2 .

approximate measure of the spread of the peaks.

\section{A. Sensitivity study}

In order to quantify the effects of various inverted parameters on the group speed values, a sensitivity analysis was performed, the results of which are tabulated in Table II. Parameters whose effects were studied include water depth, sound speed variations in the water column, and changes in sediment compressional speed in the top $30 \mathrm{~m}$ of the sediment. Values shown in the Table II are percentage increases in the value of the fitness function or objective function [Eq. (1)] due to changes in these parameters. The changes were compared with the fitness of the baseline model which corresponds to the best parameter set. Variations in the sound speeds in the water column produce considerable changes in the group speeds. The variations in the ocean sound speed along the propagation path are of the order of $5 \mathrm{~m} / \mathrm{s}$ (Fig. 10). Hence changes in fitness due to ocean sound speed variations of magnitude 5 and $3 \mathrm{~m} / \mathrm{s}$ are considered. A uniform increase of $5 \mathrm{~m} / \mathrm{s}$ in sound speed in the water column changes the fitness by $80 \%$ while the change is $92 \%$ when it is decreased. The changes are respectively $32 \%$ and $42 \%$ when the magnitude of the variation in sound speed was decreased to $3 \mathrm{~m} / \mathrm{s}$. The effect of the absence of the warm surface layer is simulated by changing the sound speed to $1512.5 \mathrm{~m} / \mathrm{s}$ from $1502.5 \mathrm{~m} / \mathrm{s}$ in the top $15 \mathrm{~m}$. This produces only $5 \%$ change in the fitness. Water depth variations have considerable impact on the group speeds as seen from the changes in the fitness. An increase in water depth by $4 \mathrm{~m}$ changes the fitness by $54 \%$ while a decrease by the same amount produces $60 \%$ change in fitness. Variations in the bottom compressional speed by 50 and $30 \mathrm{~m} / \mathrm{s}$ in the top 30 $\mathrm{m}$ of the sediment produce changes in fitness function whose magnitudes are comparable to those produced by the ocean sound speed variations ( 5 and $3 \mathrm{~m} / \mathrm{s}$ ) discussed earlier. But the natures of these changes are considerably different. The variations in the sediment speeds tend to affect the low fre- 
TABLE II. Results of the sensitivity study. Changes in the fitness are listed as percentage changes from the fitness of the baseline model.

\begin{tabular}{lcc}
\hline \hline \multicolumn{1}{c}{ Parameter } & Amount & $\begin{array}{c}\text { Change } \\
\text { in fitness } \\
\text { (percentage) }\end{array}$ \\
\hline $\begin{array}{l}\text { Sound speed in water } \\
\text { column }\end{array}$ & $+5 \mathrm{~m} / \mathrm{s}$ & 80 \\
& $+3 \mathrm{~m} / \mathrm{s}$ & 32 \\
& $-5 \mathrm{~m} / \mathrm{s}$ & 92 \\
& $-3 \mathrm{~m} / \mathrm{s}$ & 42 \\
& constant sound speed & 5 \\
Range dependent & m/s) in top $15 \mathrm{~m}$ & 12 \\
(adiabatic) & & 54 \\
Water depth & $+4 \mathrm{~m}$ & 60 \\
Sound speed in top $30 \mathrm{~m}$ & $-4 \mathrm{~m}$ & 23 \\
of sediment & $-30 \mathrm{~m}$ & 81 \\
& & 42 \\
& $+50 \mathrm{~m}$ & 82 \\
\hline \hline
\end{tabular}

quencies of higher modes to a greater extent whereas changes in water column sound speeds affect the higher frequencies of lower modes (Figs. 11 and 12).

Figure 6 shows the changes in group speeds when rangedependent analysis using adiabatic theory was applied. Sound speed profiles at eight locations along the propagation path were used to calculate the group speeds. These sound speed profiles were calculated using data collected by SeaSoar. Sediment properties were assumed range independent. The eigenvalues and eigenfunctions were assumed to vary linearly within the range sections. Group speed values were calculated at fixed intervals along the propagation path using these eigenvalues and eigenvectors and range averaged to get the mean group speed value. Differences between rangeindependent and adiabatic calculations are notable for modes 1 and 2 at higher frequencies. For higher modes, changes are minimal. Since higher modes contain more information for sediment inversion, use of range-independent analysis can be justified. This sensitivity analysis helps to arrive at the fol-

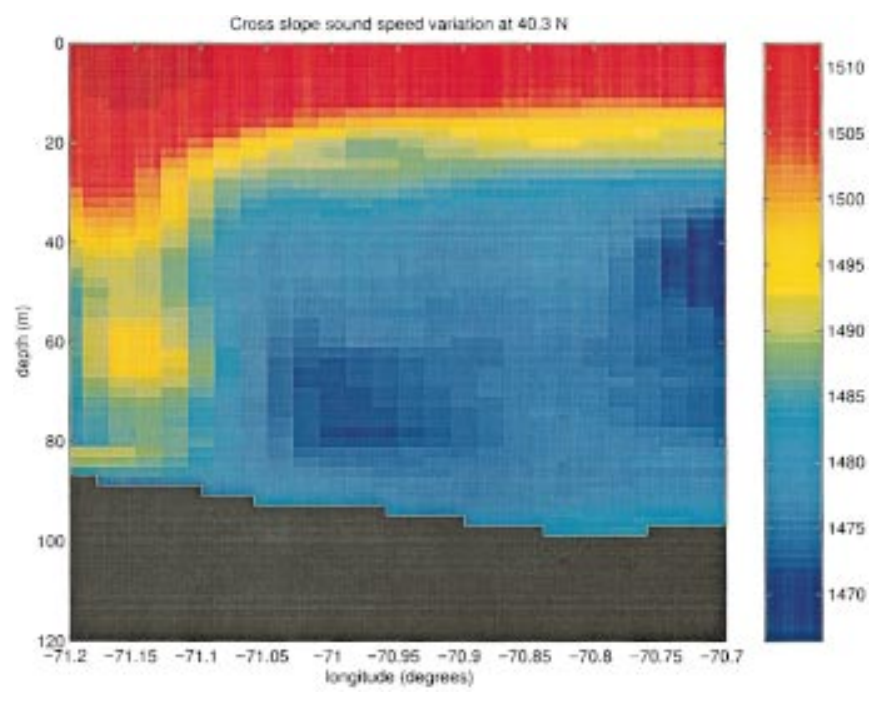

FIG. 10. Variation of sound speed along the propagation path.

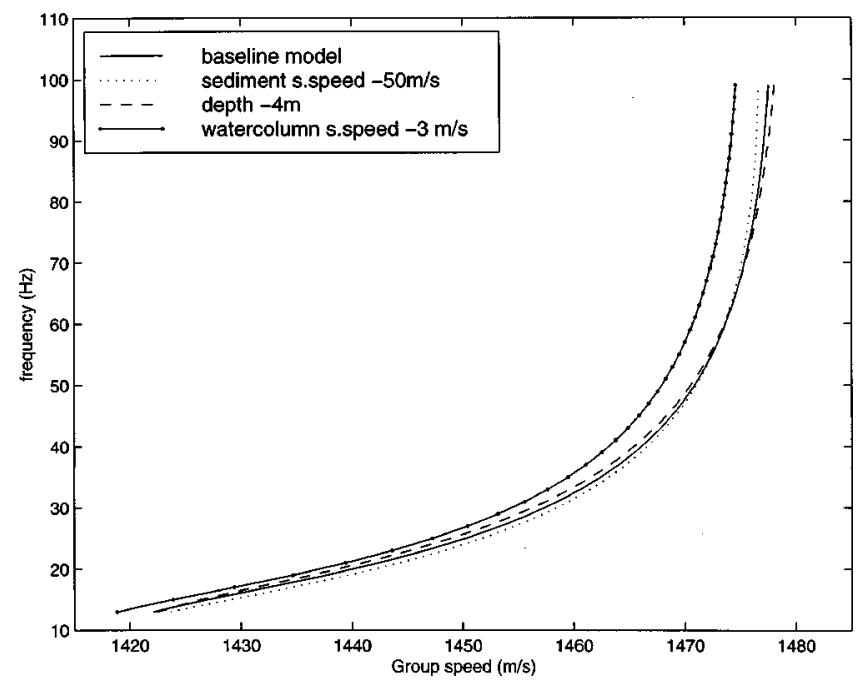

FIG. 11. Effect of changes in water depth, ocean sound speed, and sediment compressional speeds on group speeds corresponding to mode 1 .

lowing conclusions which enabled the proper formulation of the inverse problem:

(i) For the given environmental conditions existing at the present location of study, compressional sound speeds can be estimated with an accuracy of approximately $30 \mathrm{~m} / \mathrm{s}$.

(ii) Inclusion of water depth, sound speed variation in the water column, and source-receiver range will improve the quality of inversion.

(iii) Quality of data corresponding to the higher modes at lower frequencies will have greater impact on the accuracy of the sediment compressional speed values.

(iv) Range-independent inversion is appropriate for the present study.

\section{B. Genetic algorithm inversions}

Our inversion scheme discussed is based on matched field processing (MFP) concepts which encompass the following components: an environmental model, an acoustic propagation model to predict the group speed values corresponding to the unknown parameter set, an objective function which is minimized, and an efficient algorithm for searching the parameter space. The variability in the water column sound speed is modeled using empirical orthogonal functions (EOFs). The background sound speeds required to generate the EOFs were obtained from the SeaSoar measurements made at the location on the day of the SUS experiment. Figure 13 shows the EOFs used to represent the sound speed variations in the water column. The sediment is modeled as layers with unknown compressional speeds. Shear and attenuation effects are neglected for the inversion. The number of layers was fixed based on the extent of acoustic penetration into the sediment and the total number of unknown parameters that can be handled computationally. Layers are provided thin in the top $30 \mathrm{~m}$ of the sediment to take advantage of the acoustic penetration down to those depths. In addition to sound speed, water depth and source-receiver range are also treated as unknowns. Hence the unknown pa- 


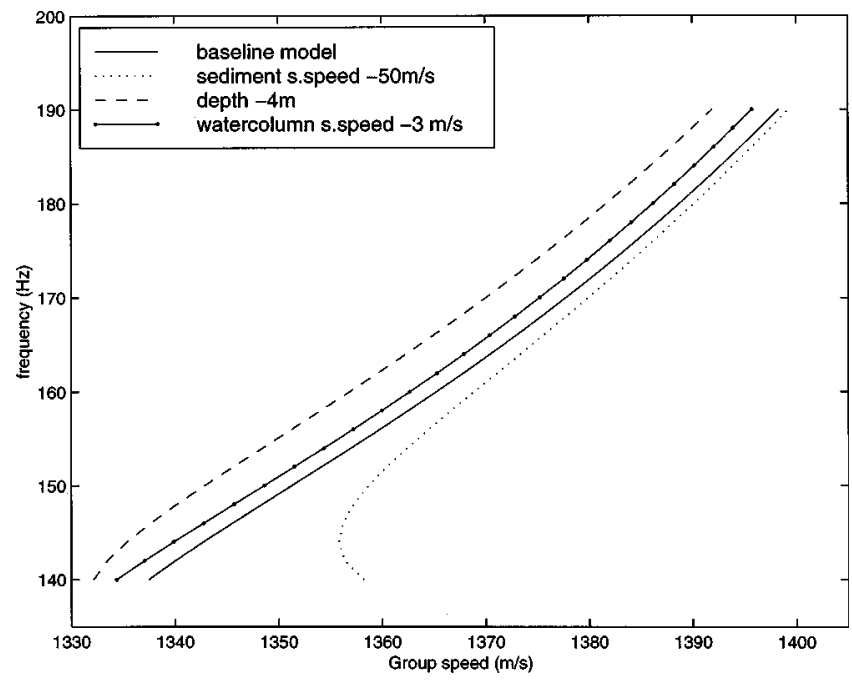

FIG. 12. Effect of changes in water depth, ocean sound speed, and sediment compressional speeds on group speeds corresponding to mode 9.

rameter set for the present study consisted of six EOF coefficients in the water column, compressional speeds in 13 sediment layers, water depth, and source-receiver range. Any a priori information about the sound speed values can be incorporated into the inversion by fixing the limits of the unknown parameter space. In the present case background information about sound speeds in the water column was available, whereas not much was known about the sediment. This necessitated a large parameter space for sediment compressional speeds. The limits of the model subspace for search varied at different depths in the present analysis. These were 1450 to $1650 \mathrm{~m} / \mathrm{s}$ at the water-sediment interface, 1600 to $1800 \mathrm{~m} / \mathrm{s}$ at $10-\mathrm{m}$ depth, and 1650 to $1850 \mathrm{~m} / \mathrm{s}$ at 30-m depth. It should also be noted that the results of the sensitivity study discussed in Sec. V A and shown in Table II directly influenced the choice of the parameters to be included as unknowns.

The fitness of each member of the population was measured based on the value of the objective function. The ob-
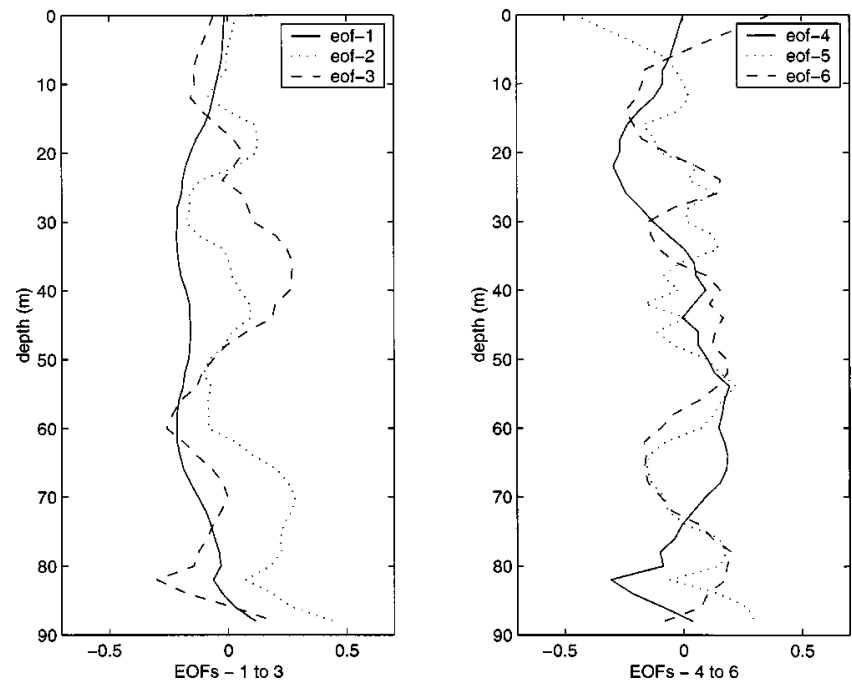

FIG. 13. The EOFs used to represent the sound speed variations in the water column. These were constructed from the background sound speed information obtained using SeaSoar observations made during the experiment.
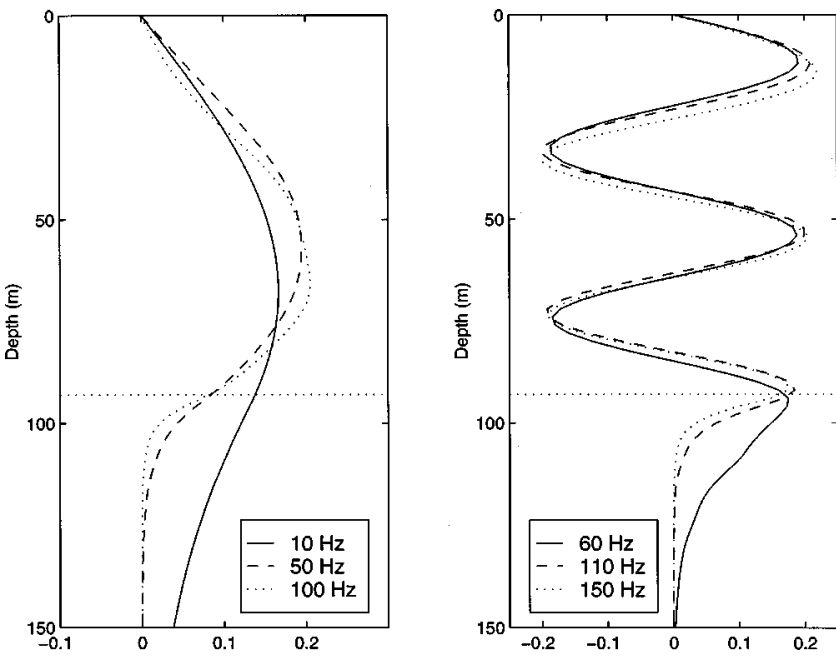

FIG. 14. Mode shapes for modes 1 and 5. The average water depth is about $93 \mathrm{~m}$.

jective function for this analysis was based on minimizing the difference between group speed values calculated using the observed time of arrivals and the predicted group speeds [Eq. (1)]. Times of arrival corresponding to frequencies in the range of $20-200 \mathrm{~Hz}$ and modes 1-9 were utilized for the inversion. Mode-formed data from all the hydrophones in the VLA were used to generate the time-frequency distributions. A range-independent normal mode routine was used to calculate the group speed values corresponding to the model vectors.

Inversions were performed using the genetic algorithm Matlab toolbox ${ }^{29}$ with a population size of 125 and with 100 generations. The stochastic universal sampling selection algorithm, real mutation and discrete recombination were adopted. Parallel GAs were run to make sure that the solution converged to the same minimum. Also, all the model parameters were stored in each generation and used to calculate the mean and covariance after assigning appropriate probability densities to them. Once the best parameter set was obtained using the GA inversion, further local optimization was done using a Levenberg-Marquardt algorithm and the linear perturbation approach. Both of these methods converged to the local minimum found near the GA inversion estimate. The perturbation inversion also gave estimates of resolution and variance.

\section{RESULTS AND DISCUSSIONS}

Mode shapes for some representative frequencies (low, intermediate, and high) are plotted in Figs. 14 and 15 for modes 1, 5, 7, and 9. These figures show the extent of the acoustic penetration into the sediments for these modes at the corresponding frequencies. These figures also help us infer the relative importance of different modes and frequencies for the inversion of sediment speeds, and the depth to which these can be estimated with reasonable accuracy. From the figures it is clear that higher modes at lower frequencies, which penetrate deep into the bottom, are relatively important for the sediment inversion. This inference is in agreement with sensitivity analysis results discussed earlier. 

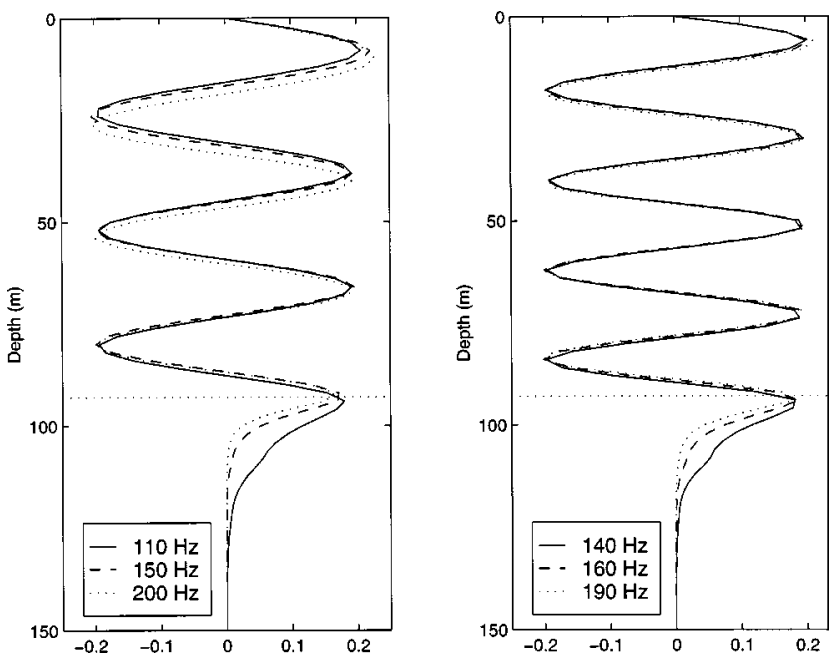

FIG. 15. Mode shapes for modes 7 and 9. The average water depth is about $93 \mathrm{~m}$.

The compressional sound speed profile obtained using GA optimization is shown in Fig. 16 for the top $40 \mathrm{~m}$ of the sediment. The three profiles shown in that figure correspond to the sound speeds calculated using the best and the mean GA parameter set and the AMCOR-6012 data. Three parallel GAs were run and the entire population from these runs was utilized to calculate the mean after weighting with appropriate probabilities based on their fitness. Also shown in the figure are the gravity core data which extend down to a depth of $1.4 \mathrm{~m}$ only.

The inversion compares reasonably well with the AMCOR sound speeds for the top $20 \mathrm{~m}$. The mean compressional speed in the upper $15 \mathrm{~m}$ of the sediment is approximately $1660 \mathrm{~m} / \mathrm{s}$, which compares favorably with the sediment compressional speeds reported by McGinnis and Otis ${ }^{23}$ and Brocher and Ewing. ${ }^{22}$ This average compressional speed corresponds to silty sands or sandy silts, ${ }^{30}$ the presence of which in the upper sediment layers has been reported by almost all the investigaters as discussed in Sec. IV A. The

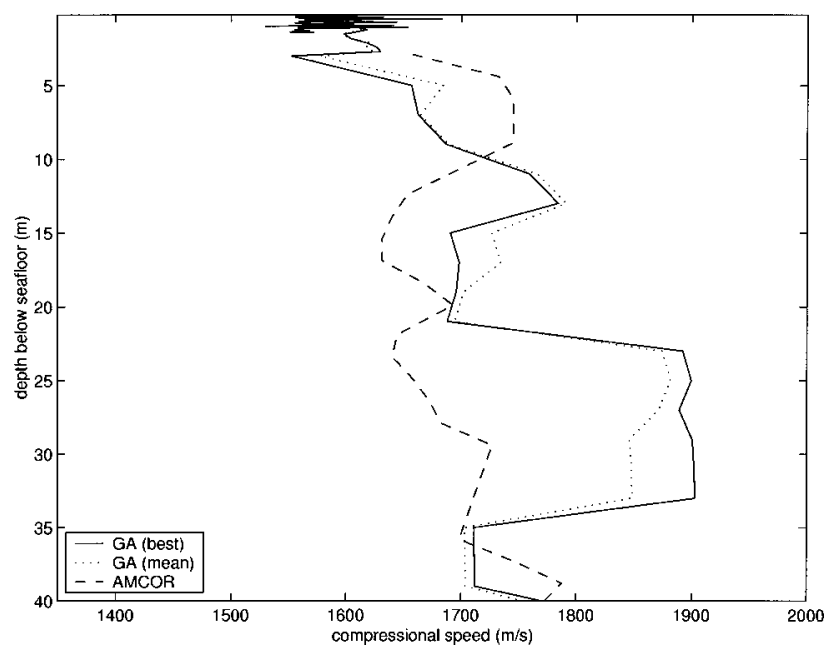

FIG. 16. Sediment compressional speeds obtained by genetic algorithm inversion. The compressional speeds obtained from the gravity cores are also shown in the top $1.4 \mathrm{~m}$ of the sediment. Compressional speeds calculated using AMCOR data are also shown. Note the difference between inversion and AMCOR at 3-7-m depth.

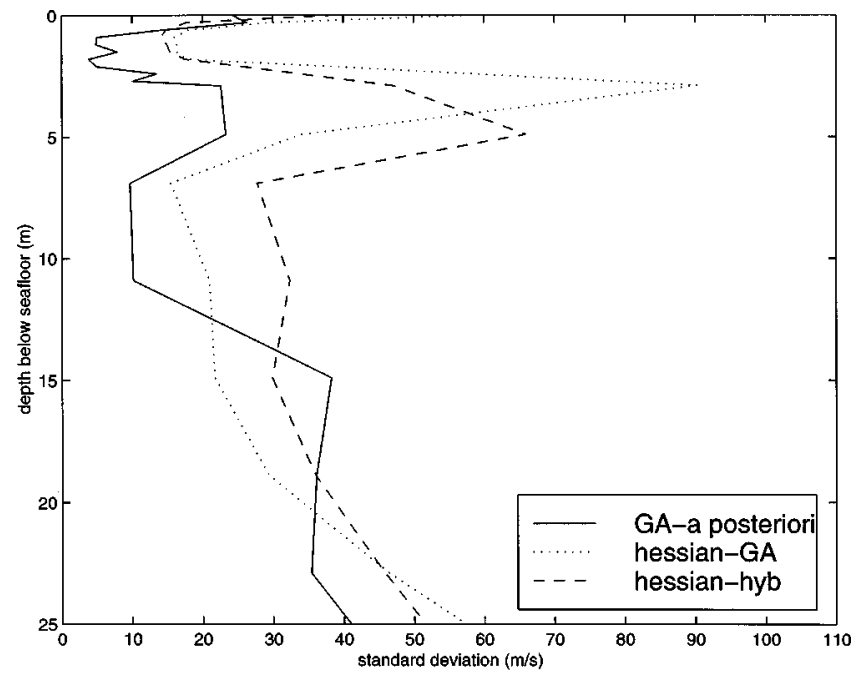

FIG. 17. Standard deviation estimates for inversions. Standard deviations shown are computed using a posteriori analysis of GA results and Hessians. Note the reduction in standard deviation due to local optimization in the top $5 \mathrm{~m}$.

high compressional speeds at $10 \mathrm{~m}$ may be due to the presence of fine or very fine sands at these depths. Both AMCOR (6010 and 6012) profiles and inversion show this high-speed layer, but at different depths. This layer with compressional speeds of $1750 \mathrm{~m} / \mathrm{s}$ is $5 \mathrm{~m}$ deeper in the case of inversion compared to the AMCOR-6012 profile. This may be due to an increase in thickness of the surface layer in the shelf region due to the deposition of sediments. It may be noted that in the case of AMCOR-6010 this high-speed layer is still deeper compared to AMCOR-6012. The compressional speed profile corresponding to the best GA parameters follow the inversion corresponding to the mean GA parameters very closely, indicating higher convergence except at depths between 5-7 $\mathrm{m}$ and $20-30 \mathrm{~m}$. It can also be seen from Fig. 17 that the standard deviation is comparatively higher at these depths. At greater depths the fitness function becomes less sensitive to sound speed variations in the sediment layers, leading to a diverse population with slightly differing fitnesses resulting in higher standard errors and a larger difference in mean and best profiles. Penetration of acoustic energy is very low below $25 \mathrm{~m}$ into the sediment.

Figure 18 shows the comparison of inversion with gravity core data. They agree very well especially below $0.6 \mathrm{~m}$. The differences between gravity core data and inversion are generally within 25 to $30 \mathrm{~m} / \mathrm{s}$. This is reasonable since the core logger has an accuracy of 5 to $10 \mathrm{~m} / \mathrm{s}$ and standard deviations of the inversion are of the order of $15 \mathrm{~m} / \mathrm{s}$ (Fig. 19).

Figure 20 shows the comparison of the group speeds calculated based on the inversions and experimentally observed values. The group speeds corresponding to the AMCOR data are also shown in the same figure. The inversion and AMCOR data differ mainly for the higher modes at low frequencies. At these locations the inversion matches the experimental data better than the AMCOR data. Interestingly for modes 1 and 2, at higher frequencies, both AMCOR and inversion differ from the experimental data. This is more likely due to the errors in sound speeds in the water column 


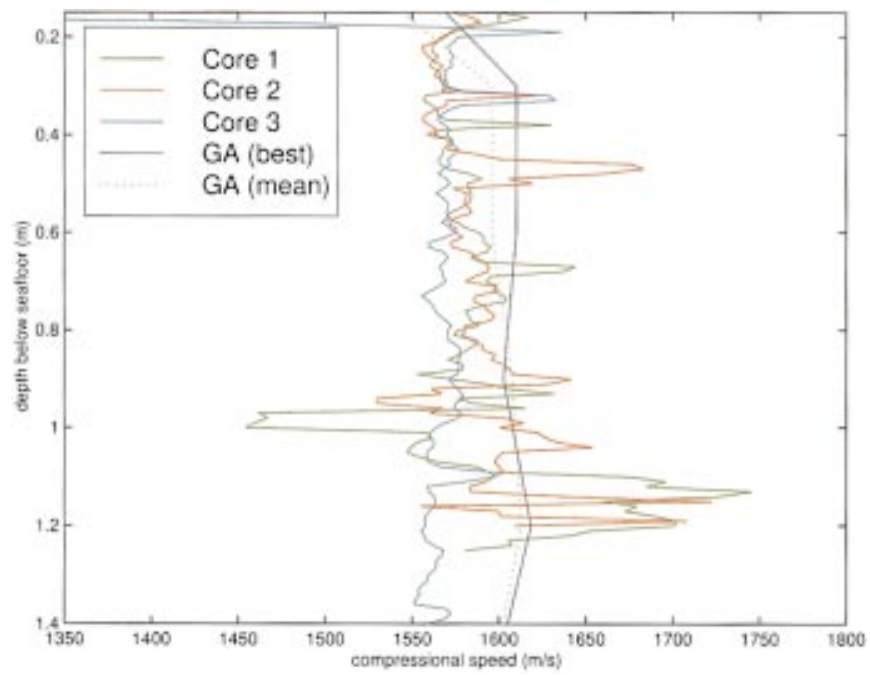

FIG. 18. Sediment compressional speeds for the top $1.4 \mathrm{~m}$ of the sediments estimated by genetic algorithm inversion. The compressional speeds obtained from the gravity cores $1-3$ are also shown.

and water depth than to errors in sediment compressional speeds.

Table III shows the EOF coefficients obtained using the inversion together with the specified search bounds. Figure 21 shows the ocean sound speed profile obtained using these EOF coefficients. Also shown in the figure are the sound speed profiles at $5-\mathrm{km}$ intervals along the propagation path. Profiles with low values at $40-\mathrm{m}$ depth correspond to the eastern side whereas those with high values at $60 \mathrm{~m}$ correspond to the western side. This cross-slope sound speed variation is shown in Fig. 10 also. Apart from the top $4 \mathrm{~m}$ of the water column, the inversion, which is a range-averaged profile, seems satisfactory. In the top $4 \mathrm{~m}$, the inversion shows a warm layer with increased sound speed. As seen previously in the sensitivity study the presence (or absence) of the warm layer does not affect the group speed values significantly. The error in the top $4 \mathrm{~m}$ might have been produced by this insensitivity. The value for the water depth was also obtained by the inversion as $92 \mathrm{~m}$ (Table III). This

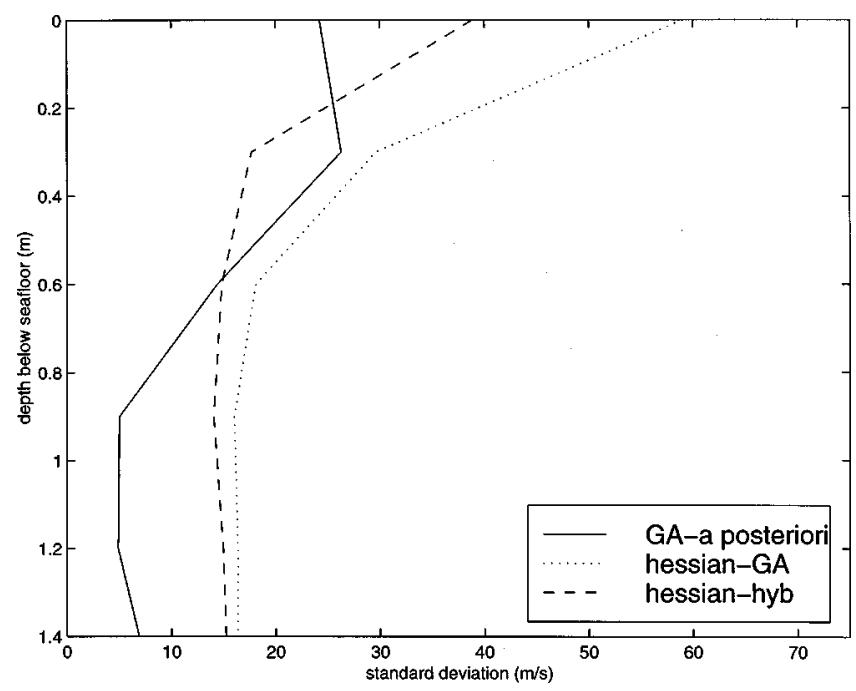

FIG. 19. Standard deviation estimates for inversions for the top $1.4 \mathrm{~m}$ of the sediments.

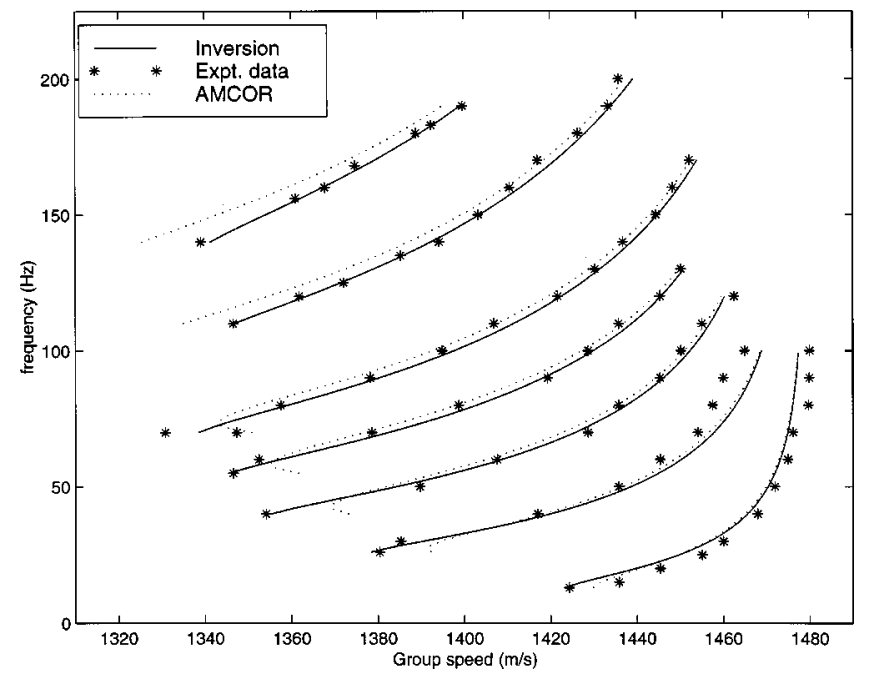

FIG. 20. Group speed dispersion curves calculated for sound speed profiles corresponding to inversion and AMCOR data. The experimental data are the group speeds estimated from mode arrival times.

seems to be the average water depth along the propagation path from the SUS shot location to the VLA.

Figure 17 shows the standard deviations computed from the entire population samples for the three GA runs. Also shown in the same figure are the standard errors calculated using the Hessian matrix. Hessians were calculated at the location of the best parameter values by calculating the curvature of the objective function numerically. These two representations of the errors show disagreement mainly at depths 2-7 $\mathrm{m}$. The higher values of standard deviations (lower values of Hessians) at these depths indicate that the GA was not able to converge to a local minimum. This was verified using local optimization methods and linear perturbation methods. The application of these methods may, in addition to this verification, lead to further improvement in the solution. The results of the hybrid inversions consisting of the application of the Levenberg-Marquardt scheme and perturbation methods are discussed in the following sections. The magnitudes of the standard errors are approximately 30 $\mathrm{m} / \mathrm{s}$ within the top $30 \mathrm{~m}$ of the sediment, which can be considered reasonable. Standard deviations in the top $1.4 \mathrm{~m}$ of the sediment are shown in Fig. 19. The average standard deviation is approximately $15 \mathrm{~m} / \mathrm{s}$ in the top $1.4 \mathrm{~m}$.

\section{A. Hybrid inversion}

A local optimization technique, which uses Hessians and/or Jacobians to guide the search from the starting point to the nearest local minimum, was employed to "fine tune",

TABLE III. Search bounds and inversion results for EOF coefficients and water depth.

\begin{tabular}{lccc}
\hline \hline \multicolumn{1}{c}{ Parameter } & Lower bound & Upper bound & Inversion result \\
\hline EOF 1 & -80 & 80 & 7.66 \\
EOF 2 & -20 & 20 & 1.25 \\
EOF 3 & -20 & 20 & -9.85 \\
EOF 4 & -20 & 20 & 13.27 \\
EOF 5 & -20 & 20 & -15.1 \\
EOF 6 & -20 & 20 & 14.8 \\
Water depth (m) & 88 & 94 & 92 \\
\hline \hline
\end{tabular}




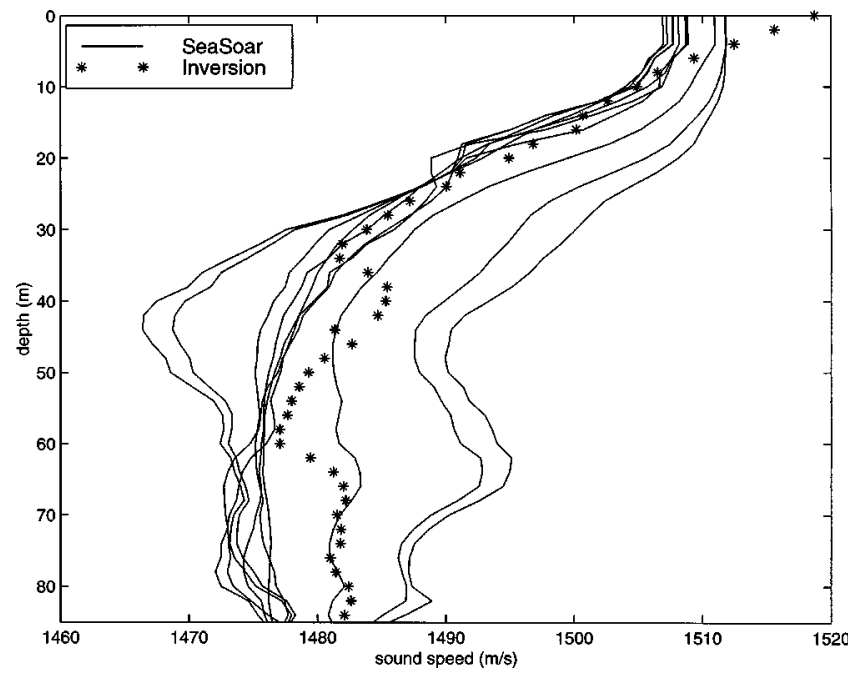

FIG. 21. Ocean sound speed profiles along the propagation path of present study. These were computed using the SeaSoar data. The sound speeds computed using the inversion are also shown.

the GA inversion results. Results of the LevenbergMarquardt inversion, using the global optimum parameters obtained from the GA inversion as starting points, are shown in Fig. 22. This hybrid inversion improves the inversion at depths 3-7 $\mathrm{m}$ and below $20 \mathrm{~m}$. This was expected as the standard deviations calculated using Hessians were comparatively large at these depths. This hybrid inversion is able to decrease the standard deviations at these depths as shown in Fig. 17. It can be noted that the velocity gradients at 5-m depth for hybrid inversion compares well with the AMCOR data. The results of the perturbation inversion using GA results as the background are shown in Fig. 23. The result of the hybrid inversion is also shown in this figure for comparison as both linear perturbation approach and the LevenbergMarquardt scheme are used to "fine tune" the GA results. The changes in the compressional speeds predicted by both these methods show a similar trend. Both these methods predict similar compressional speeds between 3-7-m depths

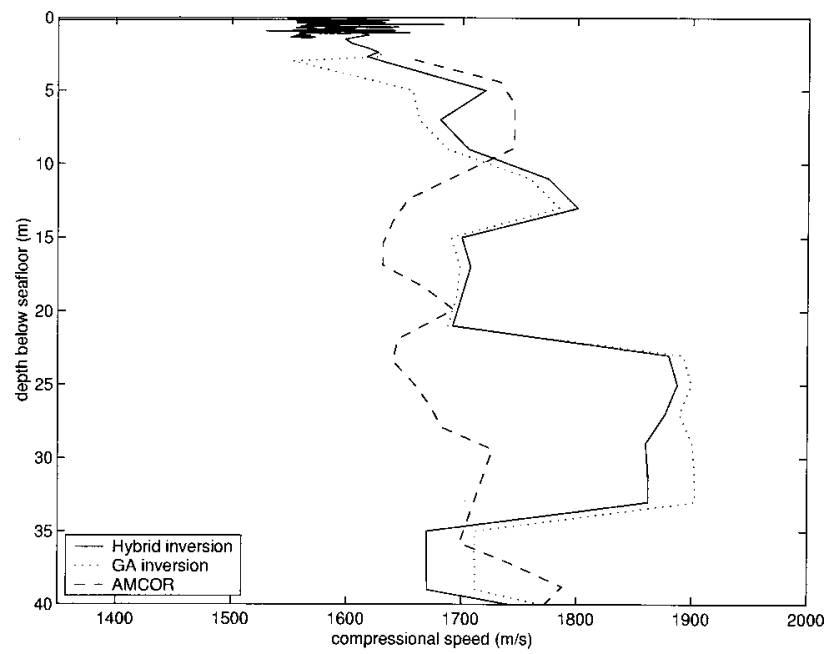

FIG. 22. Sediment compressional speeds obtained using Hybrid inversion. The Levenberg-Marquadt scheme was applied to the best parameter set produced by GA. Note the agreement between the Hybrid inversion and AMCOR data between depths 3 to $7 \mathrm{~m}$. The compressional speed obtained from the gravity cores are also shown in the top $1.4 \mathrm{~m}$ of the sediment.

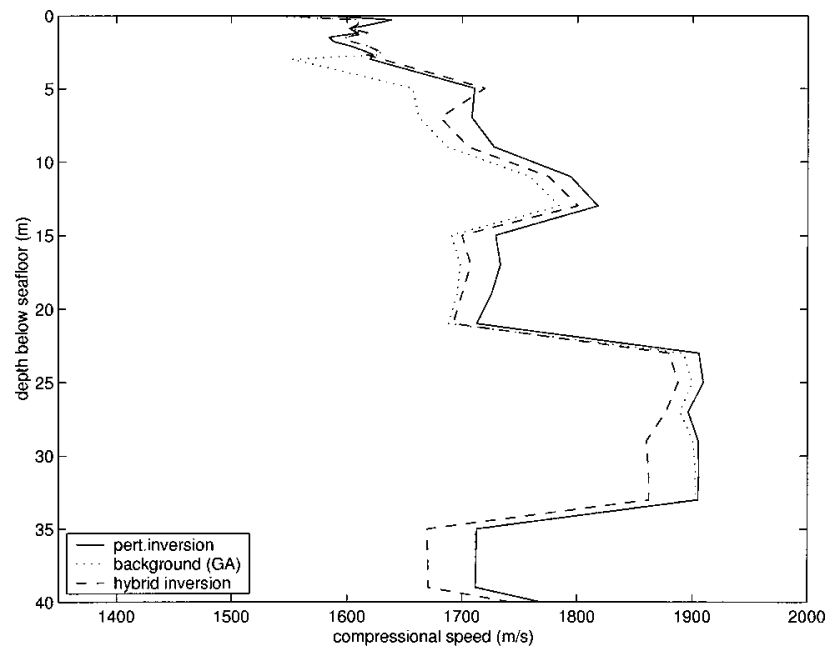

FIG. 23. Sediment compressional sound speeds obtained by linear perturbation inversion. The compressional speeds obtained by GA were used as the background profile for this inversion.

where the GA inversion is most uncertain. We can extract the standard deviation and resolution lengths from perturbation theory as shown in Figs. 24 and 25. The resolution length varies approximately from $5 \mathrm{~m} / \mathrm{s}$ at the top to $30 \mathrm{~m} / \mathrm{s}$ at $30-\mathrm{m}$ depth. The standard error is low at $6 \mathrm{~m} / \mathrm{s}$. The low values obtained may be due to the good quality of the background profile used for inversion. It should be noted that the linear perturbation inversion was carried out using weighted data as discussed in Sec. II A. The detailed variations in the values of standard deviation depthwise are likely to be caused by different modes turning at different depths.

\section{CONCLUSIONS}

Sediment compressional speeds were evaluated using hybrid optimization schemes based on the group speed dispersion properties of the shallow water waveguide. Data for the inversion were obtained from the signals produced by explosive sources. Time-frequency analysis was done using wavelets which provided better frequency-time resolution than usual Fourier-based methods. Results of the inversion provided compressional speeds of sediments down to $30 \mathrm{~m}$

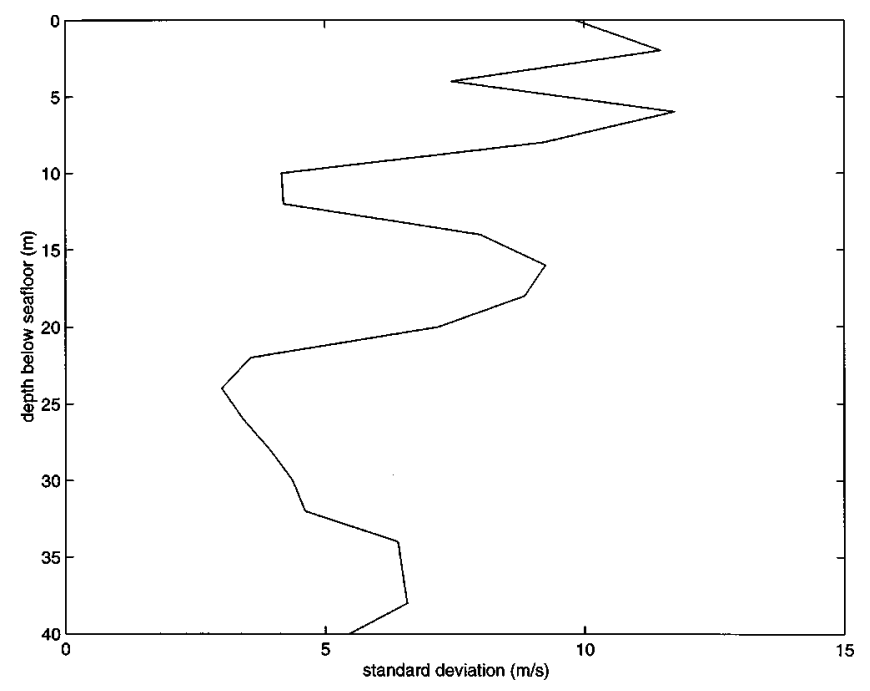

FIG. 24. Standard deviation estimates for linear perturbation inversion. 


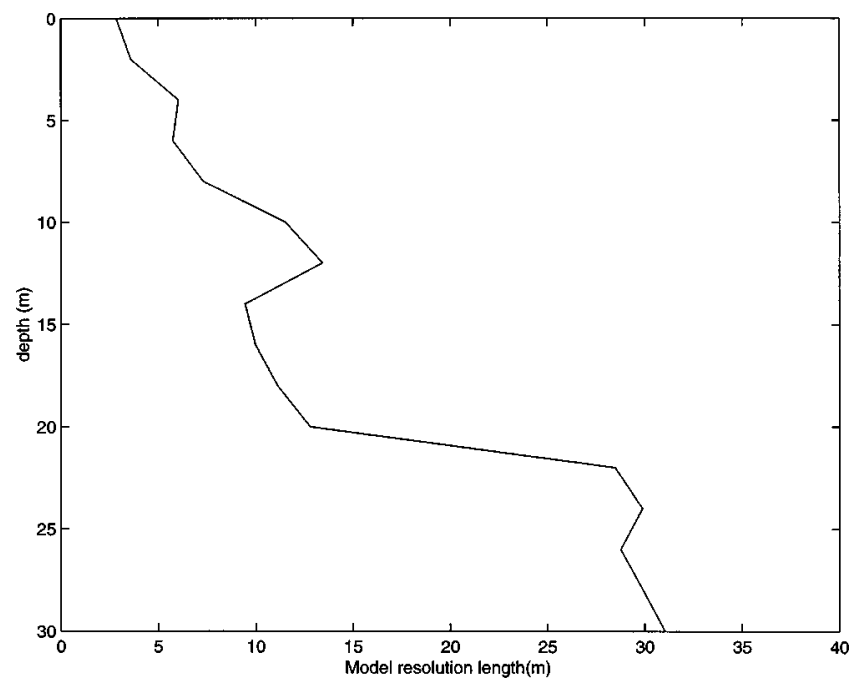

FIG. 25. Resolution length for linear perturbation inversion.

in depth. The inversion compared well to gravity core data taken at the location on the track and also to AMCOR drill site data in the top $20 \mathrm{~m}$. The calculated standard deviations for these depths were of the order of $20 \mathrm{~m} / \mathrm{s}$. The results of the sensitivity study quantified the effects of parameters of influence (sediment compressional speeds, sound speed in the water column, water depth, etc.) on the group speed values. Higher modes seem to have greater influence on the inversion of sediment properties than lower modes. This factor emphasizes the importance of obtaining good timefrequency resolution for higher modes. Efforts are currently underway to address this aspect. Also, these inversion results correspond to a range-independent environment. Extension of this scheme to range-dependent environments needs further study.

\section{ACKNOWLEDGMENTS}

The authors wish to thank the scientific team of the PRIMER experiment including Robert Beardsley, Ken Brink, Ching-Sang Chiu, Glen Gawarkiewicz, Robert Pickart, Alan Robinson, and Brian Sperry, captain and crew of R/V ENDEAVOR, and pilots and crew from Naval Air Warfare Center at Patuxent River NAS for their significant contributions to data collection. In particular, the authors recognize David Volak and Thomas Gabrielson for their contribution to experimental design. This work was supported by Office of Naval Research, Jeffrey Simmen, Code 321 OA Program Manager, and Steven Ramp, Code 322 PO Program Manager.

\footnotetext{
${ }^{1}$ A. Tolstoy, O. Diachok, and L. N. Frazer, "Acoustic tomography via matched field processing," J. Acoust. Soc. Am. 89, 1119-1127 (1991).

${ }^{2}$ P. Ratilal, P. Gerstoft, J. T. Goh, and K. P. Yeo, "Inversion of pressure data on a vertical array for seafloor geoacoustics properties," J. Comput. Acoust. 6, 269-289 (1998).

${ }^{3}$ K. B. Smith, J. G. Rojas, J. H. Miller, and G. Potty, "Geoacoustic inversions in shallow water using direct methods and genetic algorithm techniques," in Proc. Pacific Ocean Remote Sensing Conf. (PORSEC98), edited by M.-X. He and G. Chen (invited paper) (1998), pp. 703-707.

${ }^{4}$ B. Rapids, T. Nye, and T. Yamamoto, "Pilot experiment for the acquisition of marine sediment properties via small scale tomography system,' J. Acoust. Soc. Am. 103, 212-224 (1998).
}

${ }^{5}$ J. F. Lynch, S. D. Rajan, and G. V. Frisk, “A comparison of broad band and narrow band modal inversions for bottom geoacoustic properties at a site near Corpus Christi, Texas,', J. Acoust. Soc. Am. 89, 648-665 (1991).

${ }^{6}$ C. J. Pike, "Analysis of high resolution marine seismic data using wavelet transform,', in Wavelets in Geophysics, edited by E. F. Georgiou and P. Kumar (Academic, New York, 1994), pp. 1-43.

${ }^{7}$ M. D. Collins and W. A. Kuperman, "Focalization: Environmental focusing and source localization,’’ J. Acoust. Soc. Am. 90, 1410-1422 (1991).

${ }^{8}$ J.-P. Hermand and P. Gerstoft, "Inversion of Broadband Multitone Acoustic Data from the YELLOW SHARK Summer Experiments,', IEEE J. Ocean Eng. 21(4), 324-346 (1996).

${ }^{9} \mathrm{P}$. Gerstoft, "Inversion of seismoacoustic data using genetic algorithms and a posteriori probability distributions,', J. Acoust. Soc. Am. 95, 770781 (1994).

${ }^{10}$ K. S. Tang, K. F. Man, S. Kwong, and Q. He, 'Genetic Algorithms and Their Applications,'” IEEE Signal Process. Mag. 13(6), 22-37 (1996).

${ }^{11} \mathrm{P}$. Gerstoft, "Inversion of acoustic data using a combination of Genetic Algorithms and the Gauss-Newton approach,' J. Acoust. Soc. Am. 97, 2181-2190 (1995).

${ }^{12}$ M. I. Taroudakis and M. G. Markaki, ' On the use of matched-field processing and hybrid algorithms for vertical slice tomography,' J. Acoust. Soc. Am. 102, 885-895 (1997).

${ }^{13}$ R. L. Parker, Geophysical Inverse Theory (Princeton U.P., Princeton, NJ, 1994).

${ }^{14}$ M. K. Sen and P. L. Stoffa, "Rapid sampling of model space using genetic algorithms: examples from seismic waveform inversion,' Geophys. J. Int. 108, 281-292 (1992).

${ }^{15}$ P. Gerstoft and D. F. Gingras, "Parameter estimation using multifrequency range-dependent acoustic data in shallow water,' J. Acoust. Soc. Am. 99, 2839-2850 (1996).

${ }^{16}$ K. Koper and M. Wysession, "Modelling the Earth's Core and Lowermost Mantle with a Genetic Algorithm,', http://levee.wustl.edu/ seismology/koper/Papers/JGR96/pkp-ga.html, 1996.

${ }^{17}$ A. Newhall, K. Von der Heydt, B. Sperry, G. Gawarkiewicz, and J. Lynch, "Preliminary Acoustic and Oceanographic Observations from the Winter Primer Experiment,' Woods Hole Oceanog. Inst. Tech. Rep., WHOI-98-19 (1998).

${ }^{18}$ H. J. Knebel and E. Spiker, "Thickness and age of Surficial Sand Sheet, Baltimore Canyon Trough Area,', Bull. Am. Assoc. Pet. Geol. 61, 861871 (1977).

${ }^{19}$ J. M. Robb, J. C. Hampson, Jr., and D. C. Twichell, "Geomorphology and Sediment Stability of a Segment of the U.S. Continental Slope off New Jersey,' Science 211, 935-937 (1981).

${ }^{20}$ C. W. Poag, "Stratigraphy and Depositional Environments of Baltimore Canyon Trough,', Bull. Am. Assoc. Pet. Geol. 63, 1452-1466 (1979).

${ }^{21}$ J. C. Hathaway, C. W. Poag, P. C. Valentine, R. E. Miller, D. M. Schultz, F. T. Manheim, F. A. Kohout, M. H. Bothner, and D. A. Sangrey, "U.S. Geological Survey Core Drilling on the Atlantic Shelf,', Science 206, 515-527 (1979)

${ }^{22}$ T. M. Brocher and J. I. Ewing, "A comparison of high-resolution seismic methods for determining seabed velocities in shallow water,' J. Acoust. Soc. Am. 79, 286-298 (1986).

${ }^{23}$ L. D. McGinnis and R. M. Ottis, "Compressional velocities from multichannel refraction arrivals on George's Bank-northwest Atlantic Ocean,' Geophysics 44, 1022-1032 (1979).

${ }^{24}$ A. F. Richards, "Atlantic Margin Coring Project 1976-Preliminary report on shipboard and some laboratory geotechnical data,' U.S. Geol. Surv. Open File Rep., No. 78-123 (1977).

${ }^{25}$ M. Badiey, A. H-D. Cheng, and Y. Mu, "From geology to geoacousticsEvaluation of Biot-Stoll sound speed and attenuation for shallow water acoustics,', J. Acoust. Soc. Am. 95, 309-320 (1994).

${ }^{26}$ M. V. Trevorrow and T. Yamamoto, "Summary of marine sedimentary shear modulus and acoustic speed profile results using gravity wave inversion technique,' J. Acoust. Soc. Am. 90, 441-456 (1991).

${ }^{27}$ F. Auger, P. Flandrin, O. Lemoine, and P. Gonsalves, "Time-Frequency Toolbox for Matlab,' http://www.physique.ens-lyon.fr/ts/tftb.html/, 1997.

${ }^{28}$ R. J. Urick, Principles of Underwater Sound for Engineers (McGraw-Hill, New York, 1983).

${ }^{29}$ H. Pohlheim, "Genetic and Evolutionary Algorithm for use with MATLAB-version 1.83,' http://www.systemtechnik.tu-ilmenau.de/ pohlheim/GA-Toolbox/, 1996.

${ }^{30}$ E. L. Hamilton, "Geoacoustic modeling of the sea floor,' J. Acoust. Soc. Am. 68, 1313-1340 (1980). 